\title{
Acoustic Microscopy with Mechanical Scanning-A Review
}

\author{
CALVIN F. QUATE, FELLOW, IEEE, ABDULLAH ATALAR, AND H. K. WICKRAMASINGHE
}

Invited Paper

\begin{abstract}
Acoustic waves in liquids are known to have wavelengths comparable to that of visible light if the frequency is in the gigahertz range. The phenomena of Brillouin scattering in liquids is based on such waves. In helium near $2 \mathrm{~K}$ acoustic waves with a wavelength of 2000 \& were studied some ten years ago at UCLA. It follows from these observations that an imaging system based on acoustic radiation with a resolving power competitive with the optical microscope is within reach if an ideal lens free from aberrations could be found. Such a lens, which was so elusive at the beginning, is now a simple device and it is the basic component in the acoustic microscope that forms the basis for this review.

In this article we will establish the characteristic properties of this new instrument. We will review some of the simple properties of acoustic waves and show how a single spherical surface formed at a solid liquid interface can serve as this ideal lens free from aberrations and capable of producing diffraction limited beams. When this is incorporated in to a mechanical scanning system and excited with acoustic frequencies in the microwave range images can be recorded with acoustic wavelengths equal to the wavelength of visible light. We will present images that show the elastic properties of specimens selected from the fields of material science, integrated circuits, and cell biology. The information content in these images will often exceed that of the optical micrographs. In the reflection mode we illuminate the smooth surface of a crystalline material with a highly convergent acoustic beam. The reflected field is perturbed in a unique way that is determined by the elastic properties of the reflecting surface and it shows up in the phese of the reflected acoustic field. There is a distinct and characteristic response at the output when the spacing between the object and the lens is varied. This behavior in the acoustic reflection microscope provides a rather simple and direct means for monitoring the elastic parameters of a solid surface. It is easy to distinguish between different materials, to determine the layer thickness, and to display variations in the elastic constants on a microscopic scale. These features lead us to believe there is a promising future for the field of acoustic microscopy.
\end{abstract}

\section{INTRODUCTION}

I MAGING of microscopic objects with optical and electron microscopes has been closely linked to our understanding 1 of physical or biological phenomena. It is hard to conceive of a modern laboratory involved with technology or the advancement of science that is without a microscope of some kind. It has been suggested that the number of instruments in a given country used to extend our "vision" beyond the limits of the unaided eye can be used as an indicator of the progress and advancement of that region.

Electronic microstructures and electronic materials form the basic elements of solid-state electronics-electronics that underlie an ever increasing part of the complex systems that our

Manuscript received September 15, 1978; revised February 27, 1979. This research was supported in part by the Air Force Office of Scientific Research and the NBS/ARPA Program on Semiconductor Electronics.

C. F. Quate and A. Atalar are with the Edward L. Ginzton Laboratory, Stanford University, Stanford, CA 94305.

H. K. Wickramasinghe is with the Department of Electronic and Electrical Engineering, University College London, London, England. new technology makes possible. The complexity of these systems comes from an acquired ability to control material uniformity and to fabricate devices on a scale that is determined by the wavelength of light. We not only exploit this wavelength through lithography for the fabrication of devices but we also use this radiation to examine structural details with dimensions of micrometers.

The materials themselves exhibit features with those dimensions either in the form of defects in single crystals or in the alloying of two different materials to produce microscopic regions with inhomogeneous properties. Other noncrystalline materials have a grain structure which can be of this size. The strength of the material itself depends upon these microscopic features.

The entire world of biological structures is largely made up of cellular components that are microscopic in size. The elastic properties of these components are important and they dominate a large number of biomedical problems. The contractile mechanisms involved with cell movement, other contractile processes associated with muscle contraction, the deformability of cells of the blood stream, and the enormous changes in elastic properties that occur when a given cell goes through the mitotic process and divides into two separate cells are examples where the elastic properties are of primary importance.

It may seem that the tools now available for examining microscopic structures form a complete set satisfactory for every task. But there are limitations-by and large the electron microscope in the scanning version is used to examine surface features and it is restricted to objects that can withstand the environment of the vacuum chamber. The optical microscope cannot be used to examine the interior of opaque material. But there is a stronger objection to these instruments which comes from the fact that they cannot be used to examine the mechanical or elastic properties of microstructures. These elastic properties are fundamental-they make a difference as to whether the structures will hold together. The ability to examine these properties on a microscopic scale would increase our knowledge by a wide margin. The alloying spikes of aluminum and silicon, the adhesion properties of the grains that form metal alloys and ceramics, the regions surrounding dislocations and faults in single crystals that are highly stressed, the viscosity and density of intracellular material in biological cells and tissues-all of these form an enormous area in the microscopic world, an area that is worthy of study and an area where we need new tools.

This desire to examine elastic properties on a microscopic scale has motivated our work on the use of acoustic radiation in a microscope. Acoustic waves have always held a potential for microscopic imaging simply because the wavelength of sound at microwave frequencies is small. In crystals of quartz 
at low temperatures acoustic waves with a wavelength of $100 \AA$ have been generated and studied [1]. In liquid helium, wavelengths as short at $2000 \AA$ have been propagated between two parallel surfaces [2]. At room temperature in liquids the acoustic wavelengths have been much longer. Nevertheless progress to date has permitted us to work over short distances in water with wavelengths less than $1 \mu \mathrm{m}$.

Much of this article will be devoted to the use of these waves in a focused system wherein the specimen is mechanically scanned to record the image. One primary goal-a goal that has been realized within the past few months [3] -is the construction of an instrument with a resolving power equal to that of the optical instrument. This article will be devoted to a description of that work and a description of some of the micrographs that illustrate the features that can now be studied with acoustic radiation.

We will concentrate almost entirely on the version of the acoustic microscope that uses mechanical scanning and acoustic lens [4]. Before we proceed with the full description we will point out that the essential component in the system is a simple spherical lens formed at the interface between a solid such as sapphire with a high velocity of sound and a liquid such as water with a low sound velocity. There is no spherical aberration in this lens and it can be used to focus the acoustic beam into a waist with a diameter that is less than one wavelength. In water this wavelength is equal to that of visible light $(0.5$ $\mu \mathrm{m})$ for a frequency of $3 \mathrm{GHz}$. The acoustic micrographs at this frequency are beginning to approach the optical micrographs in quality.

The material in this article is confined for the most part to instruments operating above $1 \mathrm{GHz}$. This region is attractive. There we can compete with the optical microscope in resolving fine detail and we enjoy this competition. Work in the field of acoustic microscopy below $1 \mathrm{GHz}$ is important but it will not be included here since it has been covered in previous review articles [5]-[12]. The early work of Korpel [5] and the current work of Kessler [12] on laser scanning systems has established principles and pointed to areas of investigation [13] where acoustic radiation can play a unique role. The work of Tsai [14] has demonstrated the utility of acoustic waves as a method of probing bonds between opaque materials. Wilson [15] and Weglein [16] have studied integrated circuits with the acoustic microscope and they argue that increased contrast in their acoustic micrographs provides information that is not available in optical micrographs. In France, Bridoux and Torquet [17] in their work have shown that this instrument is useful for opaque objects such as fossils. Attal [18] and Wickramasinghe [19] have taught us that the phase of the output signal contains as much information about the image as does the amplitude of the signal. In Japan, Chubachi [20] has shown that piezoelectric transducers can be fabricated directly on the curved surface of the lens and thereby eliminate the spurious pulses that rattle around inside the crystal that forms the acoustic cell. In England, Bennett, Payne, and Ash [21] have demonstrated that acoustic microscopy is useful for studying the electrolytic deposition of metallic layers. In addition, this field has been discussed in several articles of a more general interest $[22]-[26]$.

\section{The Scanning Principle}

The "field of view" imaging system where the image appears either on the retina of the observer, on photographic film, or on a fluorescent screen is not a viable alternative for acoustic radiation. Other means must be found. Piezoelectric films are efficient, highly sensitive and they operate over a wide range of frequencies. These could be used to build an array of detectors in the form of an acoustic retina. In such an array careful attention would have to be given to both the phase and amplitude of the signal from each element. The degree of complexity in this system was such that we found that we were continually working on arrays. It was the microscope itself that held our interest. A single piezoelectric detector and a mechanically scanned object is an alternative. An imaging system based on scanning the beam is tightly focused and the image field is constructed point by point as the object is moved in a raster pattern through the focus of the beam.

At first we turned to this system as an expedient, but as we gained experience we found that a scanned system has advantages not found in conventional systems.

The primary drawback for the mechanical scanning system is the speed. It is slow. Several seconds are required to build up a single frame as compared to television rates of 30 frames per second. This will be overcome in time for we have built mechanical systems that operate at 10 frames per second but the work to be reported here will be limited to the systems which use slow scans.

The advantages inherent to scanning systems with focusing were not obvious in the beginning. It is now becoming evident that scanning systems which record a single point at a time exhibit properties different from those that display the entire field of view. In the scanned system there is no problem with coherent radiation. Since the energy at the focus is confined to a diameter that is less than one wavelength in dimension there are no interference fringes of the type that are common with optical microscopes that use coherent laser radiation. These fringes arise from the scattered radiation from two points on the object that are separated by many wavelengths. This point will become clear in a later section when we calculate the response of an abrupt edge for the two systems.

The scanned system is sensitive to transverse phase gradients in the object. In transmission this is an important source of contrast. In the reflection mode the highly focused beam makes it possible to study small variations in the elastic constants across the surface of a solid specimen. This turns out to be an important source of contrast in reflection images. These two points will be discussed at some length later on in this review. And finally, the sequential recording of the image point by point is ideally suited to a system that uses a microprocessor to manipulate, store, and process the image prior to display. The cost of memories is decreasing with time, and microprocessors will be important elements in future microscopes.

\section{THE FORM OF THE INSTRUMENT WITH MechanicaI SCANNING}

If acoustic imaging systems suffer from the absence of photographic film (sensitive to acoustic radiation) they have an enormous advantage (over optics) in that the wave velocity in solids can be larger than that in liquids by a factor of ten. The sketch of Fig. 1 is useful for explaining this advantage. The large velocity ratio means that there is a large angle of refraction, for a wave is traveling through the solid-liquid interface (Fig. 1(a)). This has two consequences in the microscope: 1) with a spherical interface, rays approaching from the solid will leave in a direction that is nearly radial (Fig. 1(b)), 2) with a plane interface waves approaching from the liquid will find a critical angle for total internal reflection which is much smaller than that encountered in optics (Fig. 1(c)). 


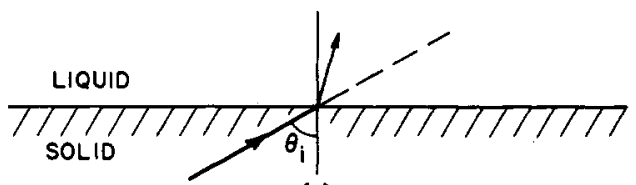

(a)

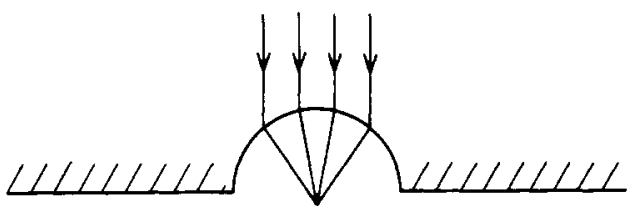

(b)

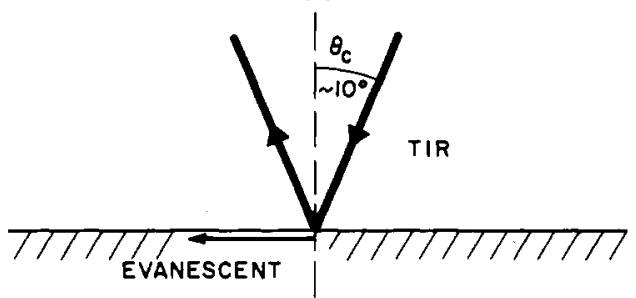

(c)

Fig. 1. Illustration of strong refraction of acoustic waves at a liquidsolid interface (TIR-total internal reflection). (a) Snell's law. (b) Lens. (c) Reflector.

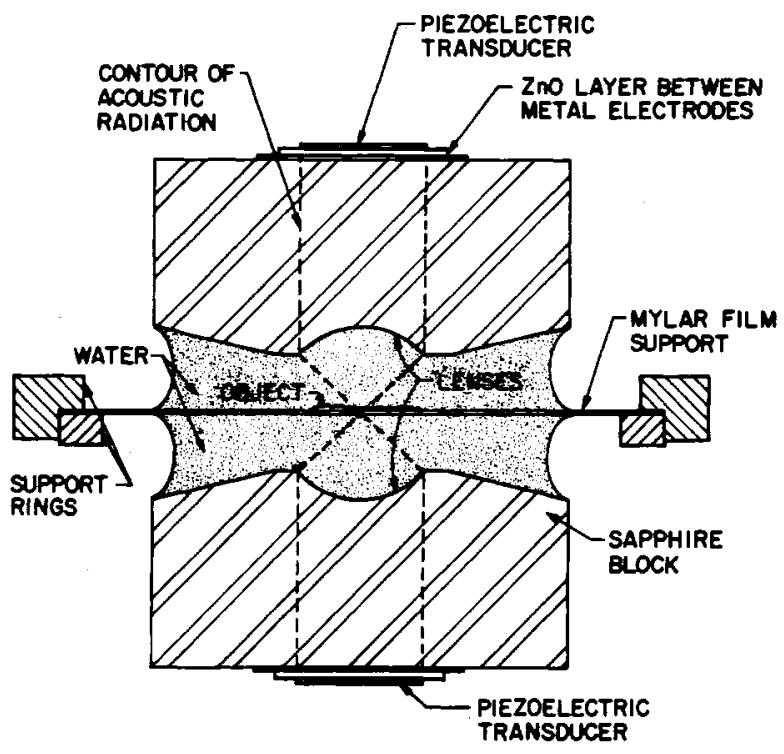

Fig. 2. Sketch of microscope geometry for transmission.

The first factor permits us to construct a simple, single surface lens that is free from aberrations and focuses the beam to a diffraction limited waist. The second factor permits us to use the reflection mode and sense the elastic properties of the reflecting surface.

The acoustic components of the microscope are shown in Fig. 2 for the transmission mode. A photograph of the mechanical components for this mode is presented in Fig. 3. The essential parts of the reflection mode microscope are shown in Fig. 4.

Referring to Fig. 2, we begin with the electrical input signal to the piezoelectric transducer. It is a sputtered film of zinc oxide sandwiched between the two films of gold. This transducer is extensively used in a variety of microwave acoustic devices [27]. It has proved to be efficient (50-percent conversion effective) and it operates at frequencies that exceed 10 $\mathrm{GHz}$. In our instrument it generates a plane wave that travels through the sapphire crystal to the spherical lens. The ratio of

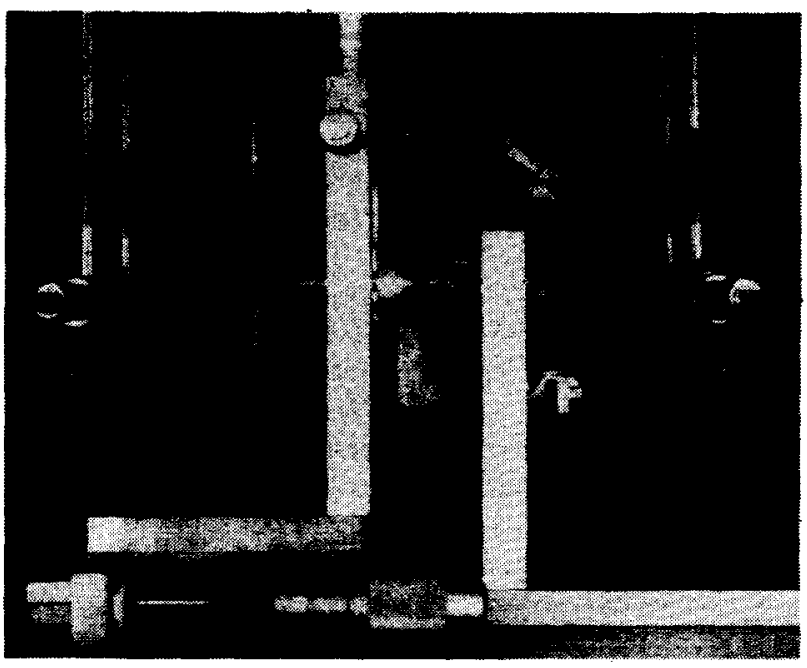

Fig. 3. Scanning acoustic microscope-Mechanical components. (Courtesy of R. C. Addison.)

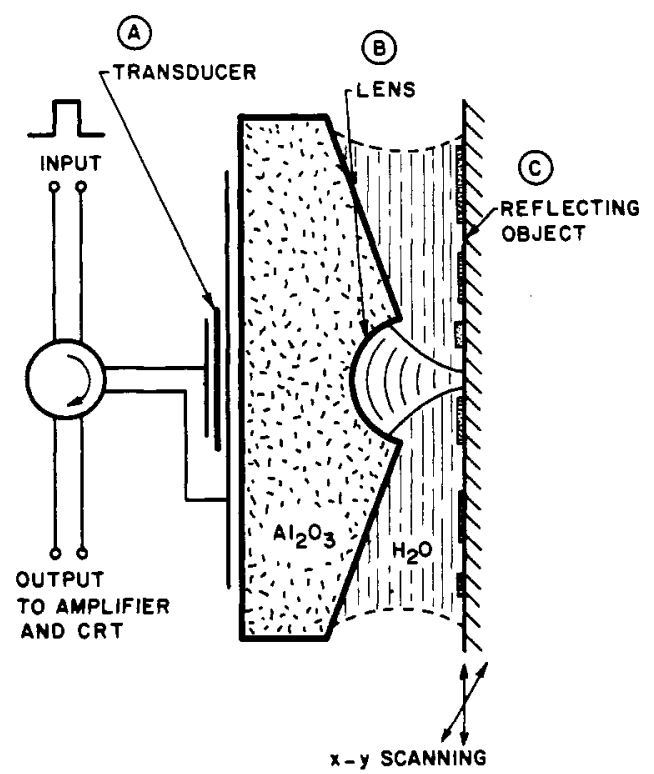

Fig. 4. Sketch of microscope components for reflection.

the radius of the transducer to that of the lens as well as the transducer-lens spacing must be carefully adjusted. The lens is in the Fresnel zone of the radiating transducer and it is surprisingly easy to pick a combination of dimensions that will result in nonuniform illuminations of the lens. At the lens surface the impedance ratio between sapphire and water is nearly $50: 1$ and this must be overcome with a quarter-wave matching layer. Various combinations have been suggestedgold-quartz [28], glass [29], Arsenic-Tri-Selenide [17]and they all are effective. Carbon films with an acoustic impedance of $9 \times 10^{6} \mathrm{~kg} / \mathrm{m}^{2}-\mathrm{s}$ may be ideal for this purpose but they have not yet been used.

With the sapphire-water combination the beam converges to a focus with a focal length that is 15 percent greater than the radius of curvature. In the transmission mode (Fig. 2) the radiation passes through the object which is supported on a thin Mylar film and it is collected by an output-lens-transducer combination that is similar to the input. In this mode where we use continuous radiation it is possible to monitor either the change in amplitude or the change in phase of the signal as the object is scanned. 


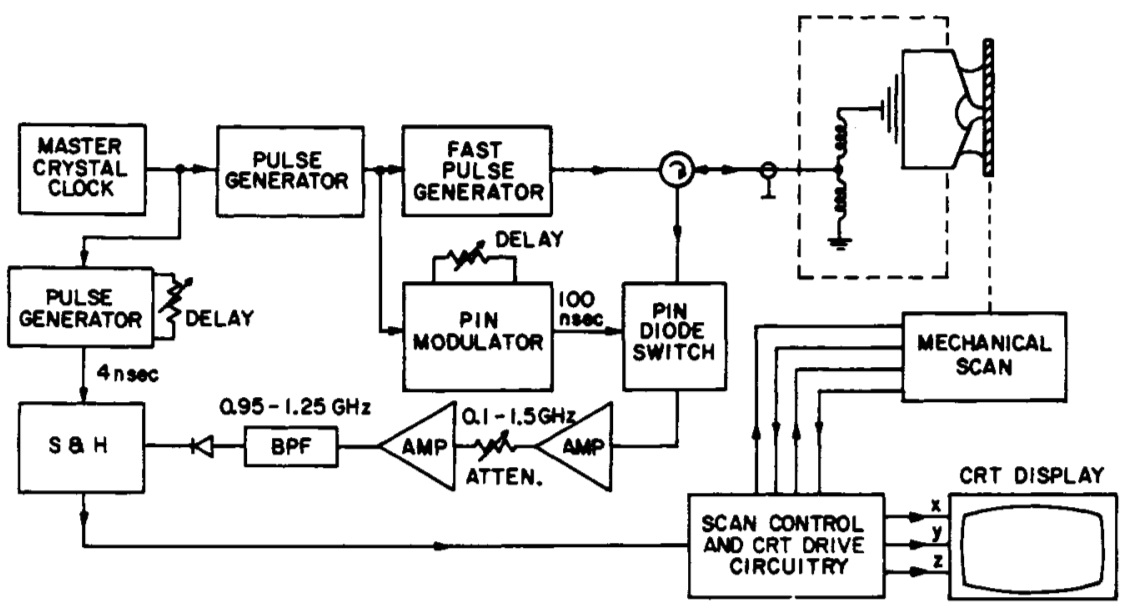

Fig. 5. Block diagram of the acoustic reflection microscope centered at $1100 \mathrm{MHz}$.

The object itself is mechanically translated through the beam waist by the loudspeaker shown in Fig. 3. This unit moves the object with simple harmonic motion along a straight line at a frequency of $60 \mathrm{~Hz}$ over a distance of $0.2 \mathrm{~mm}$. A micrometer drive is used to lift the loudspeaker through this same distance in a time equal to several seconds.

This slow scan speed (5-10 s/frame) makes it necessary to store the image by some method before display. We use two methods: 1) a scan converter, and 2) direct writing on film.

In the reflection mode (Fig. 4) the radiation is pulsed so that the reflection from the object can be distinguished in time from spurious pulses that come from other reflecting points within the system. A block diagram of the reflection mode system is shown in Fig. 5. An avalanche transistor pulser excites the piezoelectric transducer through the circulator. The pulsewidth is selected such that the frequency content of the pulse covers the operating frequency range of the acoustic system.

As an example, a pulse of duration 500 ps can excite the acoustic system centered at $1100 \mathrm{MHz}$. The return pulse will then be an RF pulse centered at $1100 \mathrm{MHz}$ and of a duration determined by the bandwidth of the acoustic transducer. After being amplified this pulse is detected and its amplitude is used to modulate the intensity ( $Z$-axis) of a television monitor. The images are recorded by photographing the face of this monitor [30].

In addition to a system with increased scan speed, other systems have been explored with features that complement and enhance those discussed here. Two are of primary interest. The nonlinear system where the output transducer is tuned to the second harmonic of the input; in this system the nonlinear properties of the object itself can be monitored [31]. In another system the axis of the output lens is moved from the colinear alignment as shown in Fig. 2 to an off-axis position [32]. It is then possible to detect the energy that is scattered through a wide angle by the object. It is analogous to dark field imaging in some ways since the output transducer does not detect the direct signal from the input when the object is absent.

\section{Properties of Acoustic Waves}

It may, at first, seem unnecessary to write down the elementary equations for acoustic waves-no one would take the space to write out the equations for electromagnetic waves-but we feel that it would be useful since acoustic wave properties do

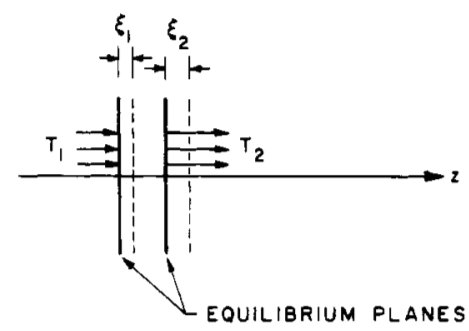

Fig. 6. Sketch of planes and then displacement from equilibrium as used in derivation of wave equation.

not have the familiar ring of dielectric constant or permeability. For that reason we will write out the differential equations. The wave equations that come from these are convenient for defining the terms that we deal with throughout the text.

Acoustic waves come in a variety of forms-shear, longitudinal, and surface-but in liquids the longitudinal wave represents the only mode of propagation. We will, therefore, limit the discussion to longitudinal, or compressional, waves where the direction of the particle motion coincides with the direction of propagation.

In the sketch of Fig. 6 we illustrate two parallel planes spaced by a distance $\Delta z$. The medium is liquid with a density $\rho_{0}$. The stress on plane 1 , denoted by $T_{1}$, and on plane 2 by $T_{2}$, represents the force per unit of area on these planes. The differential pressure, or net force, on this thin slice is $T_{1}-T_{2}$. The net force acting to compress the slice is $(-\partial T / \partial z) \Delta z$ since $T_{2}$ is given by $T_{1}+(\partial T / \partial z) \Delta z$. Compressive force will be taken as positive. The mass per unit area of this slice is $\rho_{0} \Delta z$. Newton's Force Law gives us the relation

$$
-(\partial T / \partial z) \Delta z=\rho_{0} \Delta z \partial U / \partial t
$$

or

$$
\partial T / \partial z=-\rho_{0} \partial U / \partial t .
$$

Here $U$ is the velocity of the material in the slab. It is to be distinguished from the wave $v_{s}$ velocity which will appear later. If plane 1 is displaced from its equilibrium value by an amount $\xi$ the material velocity $U$ is equal to $\partial \xi / \partial t$. If the displacement from equilibrium of plane $1, \xi_{1}$, is equal to $\xi_{2}$, the displacement of plane 2 , the slab is merely translated along the $z$-axis. But if $\xi_{2}$ is less than $\xi_{1}$ the slab is compressed by an amount $\xi_{1}-\xi_{2}=-(\partial \xi / \partial z) \Delta z$. The strain $S$ is equal to $\left(\xi_{1}-\right.$ $\left.\xi_{2}\right) / \Delta z$ or $-\partial \xi / \partial z$. If we differentiate with respect to the time 


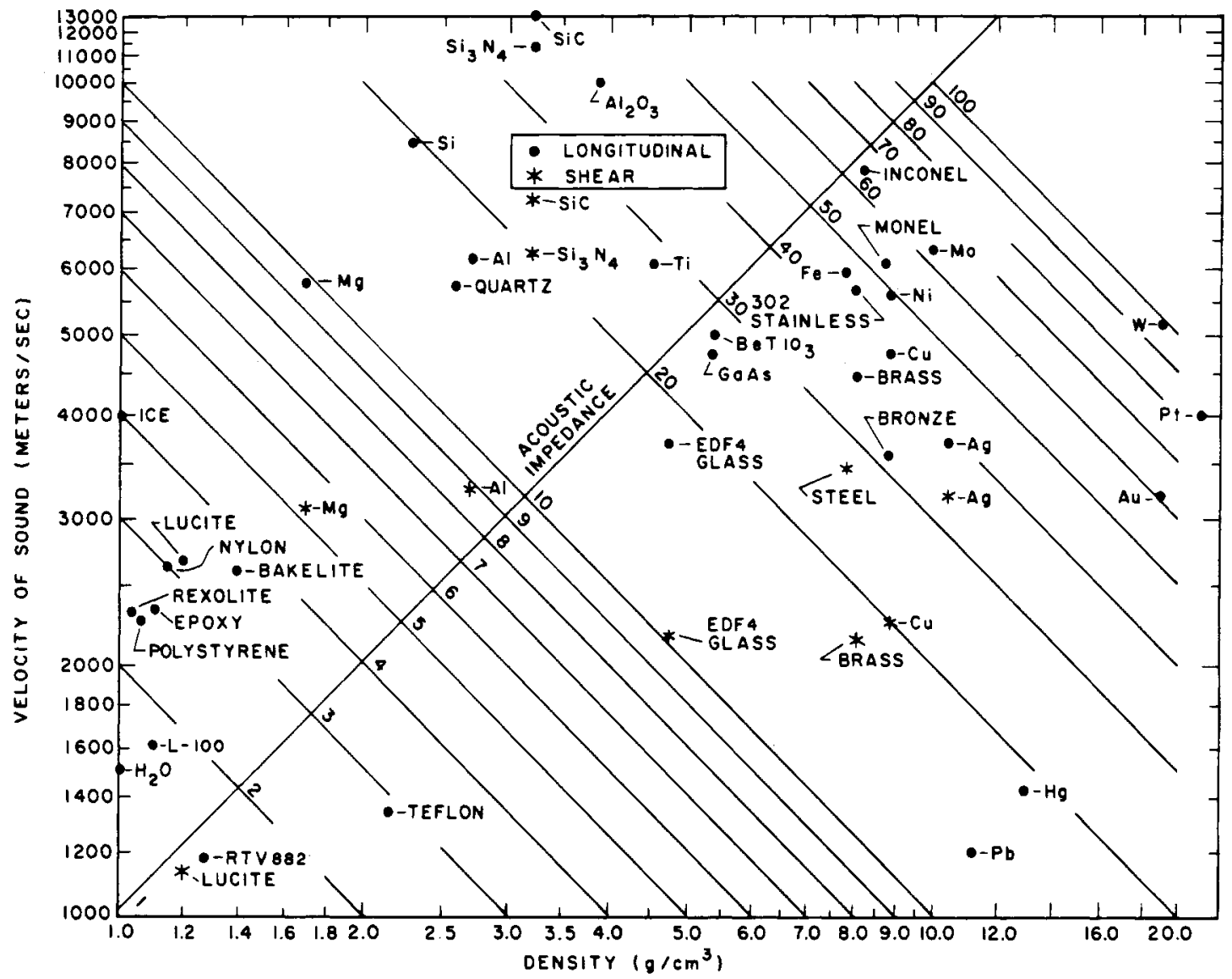

Fig. 7. Chart of material parameters for acoustic waves. (Courtesy of R. C. Eggleton.)

and invert the order of differentiation, we find

$$
\frac{\partial}{\partial t}\left(\frac{\partial \xi}{\partial z}\right)=\frac{\partial}{\partial t}(S)=-\frac{\partial U}{\partial z} .
$$

We can invoke Hooke's Law, $T=c S$, and write

$$
\partial U / \partial z=-\frac{1}{c} \partial T / \partial t
$$

Here $c$ is the elastic constant for the liquid.

The two equations (4-1) and (4-2) give us the wave equations. We assume lossless one-dimensional propagating waves in the form $\exp (-j(k z-\omega t))$ and the equations take the form

$$
\begin{aligned}
& k T=\omega \rho_{0} U \\
& k U=(\omega / c) T .
\end{aligned}
$$

We find the relation for $k$ to be of the form

$$
k= \pm \omega / \sqrt{c / \rho_{0}}= \pm \omega / v_{s}
$$

where

$$
v_{s} \equiv \sqrt{c / \rho_{0}} .
$$

With the forward wave $\left(k=+\omega / v_{s}\right)$ we have $T=+\sqrt{\rho_{0} / c} U=$ $Z_{0} U$ and with the backward wave $\left(k=-\omega / v_{s}\right)$ we have $T=$ $-Z_{0} U$.

Here $Z_{0}\left(\equiv \sqrt{c \rho_{0}}\right)$ is the characteristic impedance of the acoustic wave. It is related to the wave velocity through the relation $Z_{0}=\rho_{0} v_{s}$. These three parameters are conveniently plotted in the form as given in Fig. 7. There we see that the wave velocity $(\mathrm{m} / \mathrm{s})$ and acoustic impedance $\left(\mathrm{kg} / \mathrm{m}^{2}-\mathrm{s}\right)$ for different materials can vary by more than a factor of ten. This large variation underlines the crucial difference between acoustic imaging and optical imaging. The index of refraction, which determines both the wave velocity and wave impedance for optical waves, varies by less than a factor of two from materialto-material. It is for this reason the biological material exhibits a small contrast for optical waves and a large contrast for acoustic waves.

The similarity of (4-1) and (4-2) with the one-dimensional form of electromagnetic waves means that we can take over those solutions directly. The power flow for acoustic waves is given by

$$
P=\frac{1}{2} T U^{*}=\frac{1}{2} \frac{|T|^{2}}{Z_{0}} .
$$

The reflectivity of a wave at an interface by two materials of impedance $Z_{01}$ and $Z_{02}$ is given by

$$
\operatorname{Ref}=\frac{Z_{02}-Z_{01}}{Z_{02}+Z_{01}}
$$

and the transmission (amplitude) through the interface is given by

$$
\text { Trans }=\frac{2 Z_{01}}{Z_{01}+Z_{02}} .
$$

It also follows that the reflection at an interface can be reduced and the transmission improved with a matching layer one quarter wave in thickness with an impedance equal to $\left(Z_{01} Z_{02}\right)^{1 / 2}$. It thus forms an acoustic antireflection coating [29].

The attenuation of these waves is included by rewriting the propagation in the form $\exp (+j(k z-\omega t)) \exp (-\alpha Z)$ where $\alpha$ is the attenuation coefficient. This parameter has a strong 
TABLE I

Comparison of Calculated and Experimental Values of AtTENUATION at $1 \mathrm{GHz}$ (From WaUK [34])

\begin{tabular}{|c|c|c|c|c|c|c|}
\hline \multirow{2}{*}{$\begin{array}{l}\text { Material } \\
\text { Metals } \\
\end{array}$} & \multirow{2}{*}{$\begin{array}{c}\begin{array}{c}\text { Acoustic } \\
\text { velocity } \\
v\end{array} \\
10^{5} \mathrm{~cm} / \mathrm{sec}\end{array}$} & \multirow{2}{*}{$\begin{array}{c}\begin{array}{c}\text { Thermoelastic } \\
\text { Attenuation } \\
a\end{array} \\
\mathrm{~dB} / \mathrm{cm}\end{array}$} & \multicolumn{2}{|c|}{$\begin{array}{c}\text { Akhieser } \\
\text { Attenuation } \\
\alpha\end{array}$} & \multicolumn{2}{|c|}{$\begin{array}{c}\text { Experimental } \\
\text { Loss } \\
\alpha \\
\mathrm{dB} / \mathrm{cm}\end{array}$} \\
\hline & & & $\begin{array}{l}\mathrm{dB} \\
\text { min. }\end{array}$ & $\mathrm{cm}$ & $\begin{array}{c}a \\
1 \\
\mathrm{GHz}\end{array}$ & $\begin{array}{l}\alpha / \mathrm{f}^{2} \\
\text { (GHz) }\end{array}$ \\
\hline A1 & 6.4 & 20.5 & & & & 75 \\
\hline $\mathrm{Cu}$ & 4.4 & 42 & & & & 270 \\
\hline Au & 3.48 & 167 & - & 88 & & 200 \\
\hline $\mathrm{Pb}$ & 2.07 & 340 & & & & 500 \\
\hline$N_{1}$ & 5.31 & 3.9 & & & & \\
\hline$A_{8}$ & 3.44 & 250 & & & & \\
\hline TI & 6.33 & 0.5 & & & 10 & \\
\hline$w$ & 5.15 & 2 & & & & \\
\hline Insulators & & & & & & \\
\hline $\mathrm{Ar}_{2} \mathrm{O}_{3}$ & 11.1 & 0.0015 & .20 & .80 & $0.2-$ & \\
\hline $\mathrm{S}^{10}{ }_{2}$ & 5.9 & 0.05 & 1.64 & 6.5 & 2.5 & \\
\hline $\begin{array}{c}\text { Fused Quartz } \\
\text { YIG }\end{array}$ & 7.20 & & .35 & 1.4 & $\begin{array}{c}12.1 \\
2\end{array}$ & \\
\hline YAG & 8.56 & & .16 & .64 & $\begin{array}{l}1.1 \\
0.2-\end{array}$ & \\
\hline Semlconductors & & & & & & \\
\hline GaAs & 4.73 & & 2.7 & 10.6 & 11 & \\
\hline Insb & 3.40 & & 14.8 & 59 & $\begin{array}{l}16 \\
10\end{array}$ & \\
\hline s1 & 8.43 & 1.2 & 2.35 & 9.4 & 6.5 & \\
\hline Ge & 4.92 & 1.2 & & 24 & & $\begin{array}{l}23 \\
10\end{array}$ \\
\hline
\end{tabular}

and dominant influence on acoustic imaging at microwave frequencies. Values for typical solids are shown in Table I. In solids the attenuation is moderate and does not affect the microscope in an important way. But in liquids the attenuation is the factor that determines the ultimate resolution of the microscope. It is interesting, and somewhat ironic, to learn that this parameter-utterly different from the physical factors that limit the resolving power of an optical microscopeoperates in such a way as to limit the resolving power of the acoustic instrument to a value near the optical limit.

In metals and certain other solids a major source of attenuation is due to thermoelastic heat flow [33]. A longitudinal wave has alternating regions of compression and rarefaction. Compression can produce an increase in temperatures and the flow of heat from this heated region to the cooler region at the rarefaction point represents a source of energy loss. The path length for heat flow of this sort decreases as the frequency increases. This produces a loss per cycle which increases directly as frequency, and hence the overall attenuation increases as the square of the frequency. The values of attenuation as measured by Wauk [34] for two important metals, $\mathrm{Au}$ and $\mathrm{Ti}$, are given in Fig. 8.

In insulators an important source of attenuation has been worked out by Akhieser [35]. It can be described in terms of viscous damping of the propagating sound waves due to the "phonon gas," and the phonon-phonon collisions within this gas. Those materials included in Table I show the dramatic decrease from the values for metals. Semiconductors have attenuation crefficients which are intermediate between these values.

The theory that underlies the absorption in liquids is incomplete and we must, therefore, lean heavily on the experimental data [36]. Liquids that appear suitable for the acoustic cell

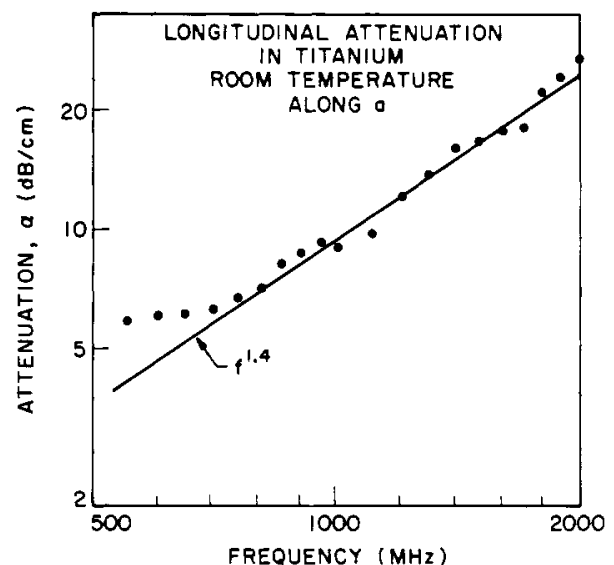

(a)

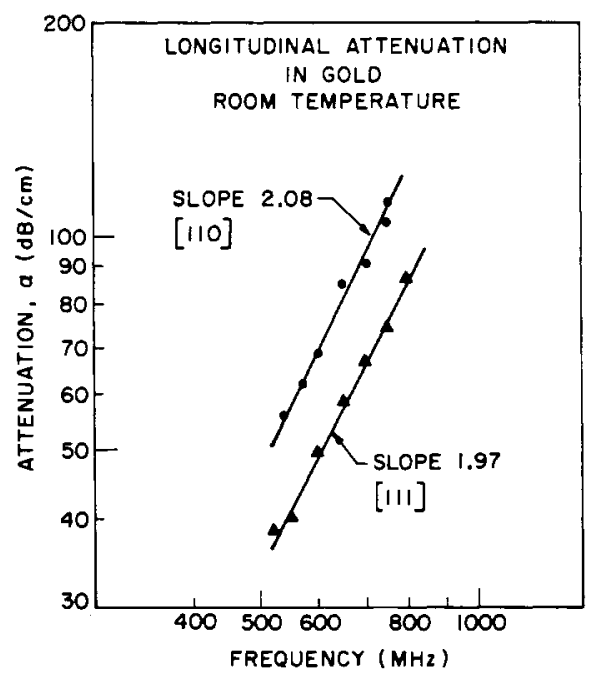

(b)

Fig. 8. Attenuation characteristics of (a) titanium and (b) gold (Wauk [34]).

can be placed into three groups-1) water where $\alpha / f^{2}$ is independent frequency (it also has a negative temperature coefficient), 2) liquids such as benzene and carbon disulphide which have a strong molecular absorption near $10^{8} \mathrm{~Hz}$ (and a positivetemperature coefficient), and 3 ) cryogenic liquids such as argon and helium.

The characteristics of water are shown in Fig. 9 where we see the reduced absorption with elevated temperature [28]. The velocity does increase slightly with heating but it is not an important effect. The high attenuation at low temperatures is attributed to a component in the liquid with an ice-like open structure. It has been found that the absorption coefficient near room temperature can be reduced by as much as a factor of two with the addition of an electrolyte [37]. It means that saline solutions can be used in connection with biological systems.

In the second category we find liquids with a lower velocity than water but a low-frequency absorption that is enormous. This high value of attenuation comes from molecular vibration near $100 \mathrm{MHz}$. For selected liquids it can be described by a single relaxation time constant $\tau$ or more conveniently by a critical frequency $f_{c}=\frac{1}{2} \pi \tau$ [36]. The absorption is frequency dependent and takes on the following form

$$
\alpha / f^{2}=B+A /\left(1+\left(f / f_{c}\right)^{2}\right) .
$$

In typical cases $A$ is three orders of magnitude larger than $B$, and it is therefore necessary to work at $f>100 f_{c}$ before we 


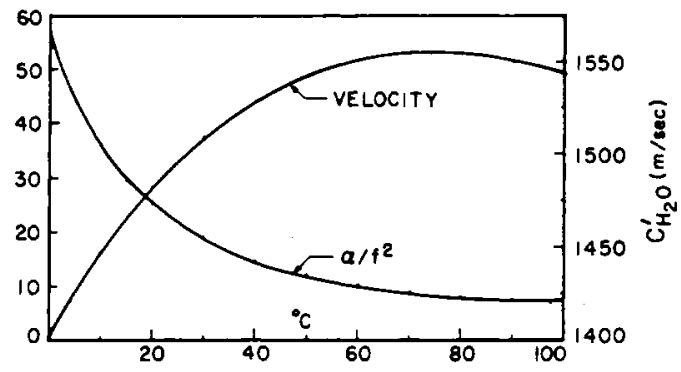

Fig. 9. Acoustic wave properties of water as a function of temperature (Lemons [28]).

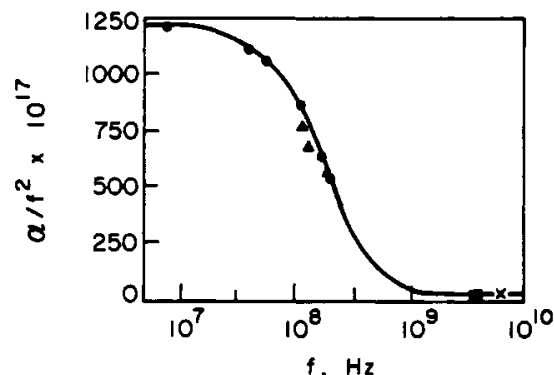

(a)

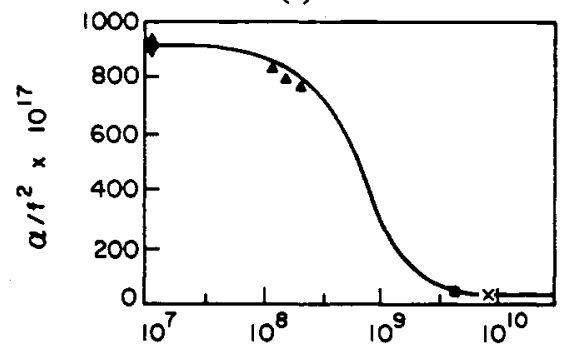

(b)

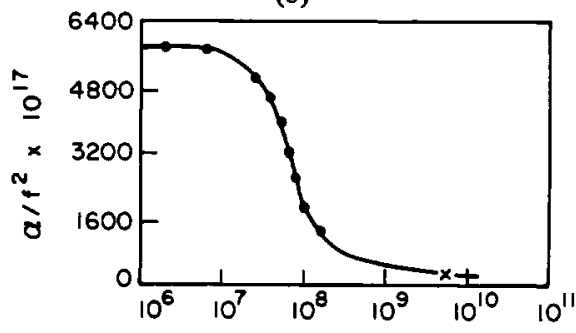

f., $\mathrm{H}$

Fig. 10. Absorption characteristics for liquid described with a single relaxation time (Fabelinskii [36]). Benzene. (c) Carbon disulfide.

can neglect the $A$ coefficient in the absorption. The experimental curves taken from the work of Fabelinskii (and reproduced in Fig. 10) [36], are typical of liquids with a single relaxation frequency. The liquid carbon disulphide is interesting at high frequencies since the attenuation and the velocity are both less than water. It is a classic example of high absorption arising from molecular vibrations. In this case it is a bending mode of the $\mathrm{CS}_{2}$ molecule which peaks near $78 \mathrm{MHz}$ [33]. In Fig. 11 we show on an enlarged scale, the comparative absorption of $\mathrm{CS}_{2}$ near $3 \mathrm{GHz}$.

These curves are significant in our work for a most interesting reason. The high-frequency limit is not known for many liquids, and for those liquids where it has been measured, the accuracy is not high. The work in liquids for frequencies greater than $4 \mathrm{GHz}$ has been carried out through Brillouin scattering of laser beams. With this technique liquids with

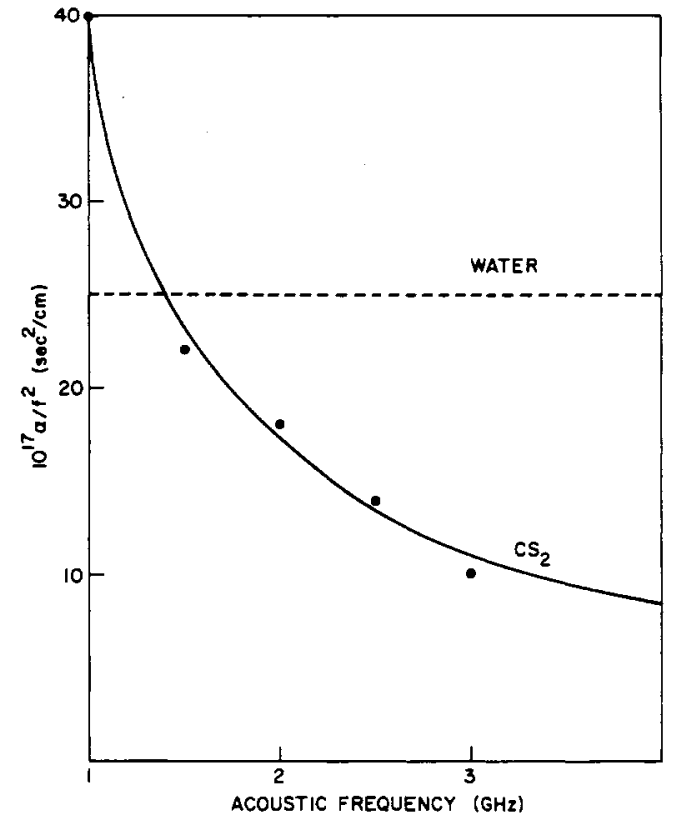

Fig. 11. Detail of absorption characteristic for carbon disulphide as compared to water (Attal [38]).

low attenuation coefficients $\left(\alpha / f^{2}<5 \times 10^{-17}\right)$ are difficult to measure since this corresponds to the limit imposed by the linewidth of the laser itself. If you were optimistic by nature (as we are!), this would suggest that more work needs to be done in this frequency range using the direct method of monitoring of absorption in a variable length acoustic cell.

At cryogenic temperatures liquids have both low attenuation and low velocity [2]. Two hold promise-argon with a velocity of $0.86 \times 10^{5} \mathrm{~cm} / \mathrm{s}$, and superfluid helium with a velocity of $0.24 \times 10^{5} \mathrm{~cm} / \mathrm{s}$. But we will not dwell on these possibilities since a discussion of operation at cryogenic temperatures is outside the scope of this review.

The examples included here offer some hope that a liquid might be found with a velocity near $10^{5} \mathrm{~cm} / \mathrm{s}$ and a value of $\alpha / f^{2}$ less than $5 \times 10^{-17} \mathrm{~s}^{2} / \mathrm{cm}$. If a liquid with these properties could be found it would permit us to operate the microscope with a wavelength of $0.25 \mu \mathrm{m}$.

\section{Converging Beams}

\section{A. Spatial Frequencies}

\section{In 1973, Cathey wrote [39]}

The impression of information onto an electromagnetic wave by modulating some parameter, such as amplitude, frequency, or phase, as a function of time is a familiar concept. Spatial information can also be impressed on the wave by amplitude, frequency, or phase modulation. ... .

He goes on in Chapter 5 to introduce the concept of spatial frequencies and he illustrates how these procedures can be used to deal with imaging problems. In earlier work, Goodman [40] and $O^{\prime} N e i l l$ [41] made extensive use of the spatial frequency concept to analyze systems for imaging. A large part of this later writing used the work of Ratcliffe [42] as a source. Ratcliffe's article is so clearly written and it sheds so much light on spatial frequency decomposition that it is still today well worth reading. Champeney [43] has discussed the comparative merits of the classical approach using diffraction integrals of this approach based on angular spectrum and spatial frequencies. 


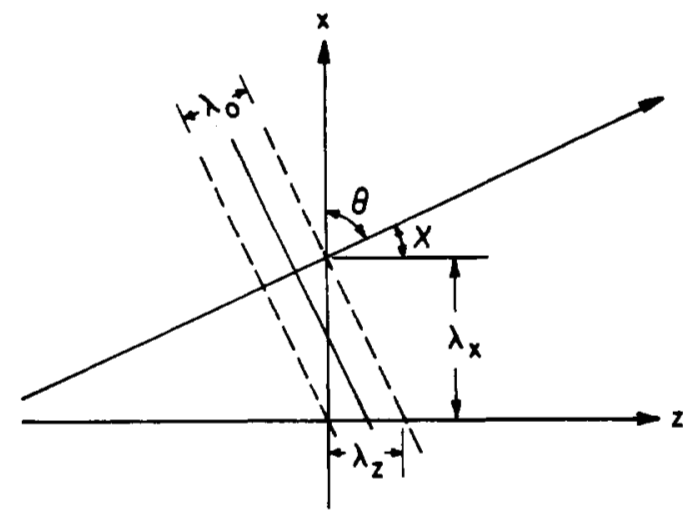

Fig. 12. Sketch for a plane wave in $x-z$ plane showing $\lambda_{x}$ and $\lambda_{z}$ as components of $\lambda_{0}$. For this wave $k_{0}=2 \pi / \lambda_{0}, k_{x}=2 \pi / \lambda_{x}=k_{0} \cos$ $\theta, k_{y}=0$, and $k_{z}=2 \pi / \lambda_{z}=k_{0} \cos \chi$. Wave equation demands that $k_{x}^{2}+k_{y}^{2}+k_{z}^{2}=k_{0}^{2}$.

The sketch of Fig. 12 shows the appropriate geometry for a plane wave propagating as

$\exp \left(-j \omega t+j k_{0} \cos \theta x+j k_{0} \cos \chi z\right)=\exp \left(-j \omega t+j k_{x} x+j k_{z} z\right)$

where $\lambda_{0}$ is the free-space wavelength and $\theta, \chi, k_{x}$, and $k_{z}$ are defined in Fig. 12. We will hereafter suppress the exponential time factor and use the following notations:

$u$ amplitude distribution of the actual function of both $x$ and $y$;

$A$ spectrum of plane waves that are used to constitute the spatial distribution $u$. It is a function of $k_{x}$ and $k_{y}$.

These two are related via the Fourier transform and this will become clear as we proceed.

We first develop the relation between the spatial distribution and the angular spectrum for a system that is uniform in the $y$ direction. We can, therefore, deal only with the $x$ and $z$ coordinates as indicated in Fig. 12. The amplitude of the plane wave components propagating at the angle $\theta$ is denoted by $A(\theta) d \theta$, and the component directed along the $z$-axis is $A(\theta) \sin \theta d \theta$. These plane waves can be summed over $\theta$ to form the spatial distribution of $u(x)$ at $z=0$

$$
u(x)=\int_{-\pi / 2}^{+\pi / 2} A(\theta) \sin \theta \exp \left(-j k_{x} x\right) d \theta
$$

We note that $k_{x}$ will serve as well to denote this component since $k_{x}=k_{0} \cos \theta$. In this treatment where we deal with a single frequency $k_{0}$ is constant and we have therefore $d k_{x}=$ $-\sin \theta d \theta k_{0}$. Equation (5-1) takes the form

$$
u(x)=-\frac{1}{k_{0}} \int_{-k_{0}}^{+k_{0}} A\left(k_{x}\right) \exp \left(-j k_{x} x\right) d k_{x} .
$$

For $k_{x}>k_{0}$ the waves at $z=0$ are evanescent and do not propagate. Nevertheless, they can be included without undue difficulty [44] and the limits can be extended to $\pm \infty$. After suitable normalization, this can be written as

$$
u(x)=\int_{-\infty}^{+\infty} A\left(k_{x}\right) \exp \left(-j k_{x} x\right) d k_{x}
$$

In this form we recognize that the spatial distribution at $z=0$ is the Fourier transform of the angular spectrum distribution $A\left(k_{x}\right)$. It follows directly from this that the angular spectrum.

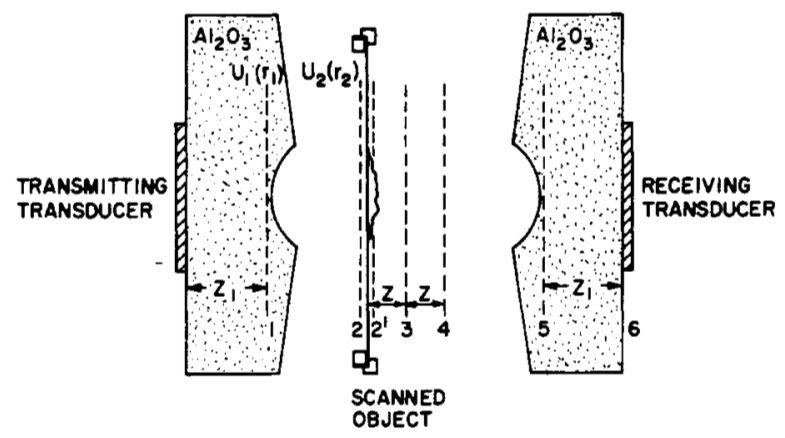

Fig. 13. Sketch of imaging system showing the various planes beginning with the input to plane 1 at the lens to plane 3 at the focal point and finally plane 6 at the output.

$A\left(k_{x}\right)$ is given by the inverse transform of $u(x)$. With a spatial distribution in both $x$ and $y$, these results can be extended to give

$$
u(x, y)=\int_{-\infty}^{+\infty} A\left(k_{x}, k_{y}\right) \exp \left[-j\left(k_{x} x+k_{y} y\right) d k_{x} d k_{y}\right]
$$

and the inverse

$$
A\left(k_{x}, k_{y}\right)=\frac{1}{(2 \pi)^{2}} \int_{-\infty}^{+\infty} u(x, y) \exp \left[+j\left(k_{x} x+k_{y} y\right)\right] d x d y
$$

In more compact notation, equation (5-3) becomes

$$
u(x, y)=\mathfrak{F}\left\{A\left(k_{x}, k_{y}\right)\right\}
$$

and equation (5-4) becomes

$$
A\left(k_{x}, k_{y}\right)=\mathfrak{F}^{-1}\{u(x, y)\} .
$$

The power of this analytical approach is evident when we consider the problem of determining the spatial distribution $u_{1}(x, y)$ across a plane 1 a distance $z_{1}$ from the input plane. We know the distribution at the input plane to be $u_{0}(x, y)$. The appropriate geometry for our complete system is sketched in Fig. 13. With the decomposition into plane waves as treated in (5-3), we can easily see the change in each of the plane waves as they move from the input plane to plane 1 . It is merely a phase shift given by

$$
A_{1}\left(k_{x}, k_{y}\right)=A_{0}\left(k_{x}, k_{y}\right) \exp \left[+j k_{z} z_{1}\right]
$$

where $k_{z}=\left(k_{0}^{2}-k_{x}^{2}-k_{y}^{2}\right)^{1 / 2}$ as indicated in Fig. 12 .

The most illustrative case and one that serves our purpose here is for a beam that is rather narrowly confined around the $z$-axis. In this case both $k_{x}$ and $k_{y}$ are small as compared to $k_{0}$ and we have

$$
k_{z} \simeq k_{0}\left(1-\frac{k_{x}^{2}+k_{y}^{2}}{2 k_{0}^{2}}\right)
$$

The relation in (5-5) then becomes

$$
A_{1}\left(k_{x}, k_{y}\right)=A_{0}\left(k_{x}, k_{y}\right) \exp \left(j k_{0} z_{1}\right) \exp \left[-j\left(\frac{k_{x}^{2}+k_{y}^{2}}{2 k_{0}}\right) z_{1}\right]
$$


where $A_{0}\left(k_{x}, k_{y}\right)$ is related to the spatial distribution at the input plane by (5-4). The spatial distribution at the output plane is written with the aid of (5-6) in the form

$$
\begin{aligned}
u_{1}(x, y)=\exp \left(j k_{0} z_{1}\right) & \int_{-\infty}^{+\infty} A_{0}\left(k_{x}, k_{y}\right) \exp \left[-j\left(\frac{k_{x}^{2}+k_{y}^{2}}{2 k_{0}}\right) z_{1}\right] \\
& \times \exp \left[-j\left(k_{x} x+k_{y} y\right)\right] d k_{x} d k_{y}
\end{aligned}
$$

or in compact notation we write

$u_{1}(x, y)=\exp \left(j k_{0} z_{1}\right) \mathcal{F}^{-1}\left\{A_{0}\left(k_{x}, k_{y}\right) \exp \left[-j\left(\frac{k_{x}^{2}+k_{y}^{2}}{2 k_{0}}\right) z_{1}\right]\right\}$.

But we know from the theorems of Fourier transformation that

$$
\mathcal{F}^{-1}\{g h\}=\mathcal{F}^{-1}\{g\} * \mathcal{F}^{-1}\{h\}
$$

and

$$
\mathcal{G}^{-1}\left\{\exp \left(-j \frac{k_{x}^{2} z}{2 k_{0}}\right)\right\}=\left(\frac{k_{0}}{2 \pi z}\right) \exp \left(-j \frac{k_{0} x^{2}}{2 z}\right) .
$$

With these we can write in place of (5-7)

$$
\begin{aligned}
u_{1}(x, y)= & \left(\frac{k_{0}}{2 \pi z}\right) \exp \left(j k_{0} z_{1}\right) \int_{-\infty}^{+\infty} u_{0}\left(x_{0}, y_{0}\right) \\
& \times \exp \left[\frac{j k_{0}\left(x-x_{0}\right)^{2}}{2 z_{1}}\right] \\
& \times \exp \left[\frac{j k_{0}\left(y-y_{0}\right)^{2}}{2 z_{1}}\right] d x_{0} d y_{0} .
\end{aligned}
$$

This permits us to move from the input plane to plane 1 just prior to the lens itself (see Fig. 13). In traversing the lens the angular spectrum is modified in phase according to the expression

$$
\exp \left[\frac{-j k_{0}\left(x^{2}+y^{2}\right)}{2 f}\right]
$$

where $f$ is the focal length of the lens (see Fig. 14). At the focal plane itself the distribution is $u_{2}(x, y)$. It is equal to Fourier transform of the spatial distribution at the lens input $u_{1}(x, y)$ as modified by the pupil function $P\left(x_{1}, y_{1}\right)$. That is we can write

$$
u_{2}(x, y)=\left(\frac{1}{j \lambda_{0} f}\right) \exp \left(j k_{0} f\right) \mathcal{F}\left\{u_{1}\left(x_{0}, y_{0}\right) P_{1}\left(x_{0}, y_{0}\right)\right\}
$$

where the transform is now limited by the pupil function to the region

$$
k_{x}= \pm \frac{k_{0} x}{f} \quad \text { and } \quad k_{y}= \pm \frac{k_{0} y}{f}
$$

In writing the pupil function in this way we have assumed that the diameter of the beam at the focal plane is much less than the radius of the lens $R$. In a generalized treatment the pupil function can include numerous interface factors such as the effect of a quarter-wave matching layer. From focal plane we can with (5-10) determine the spatial distribution at a distance $z$ from the focal plane. At that plane we encounter the object

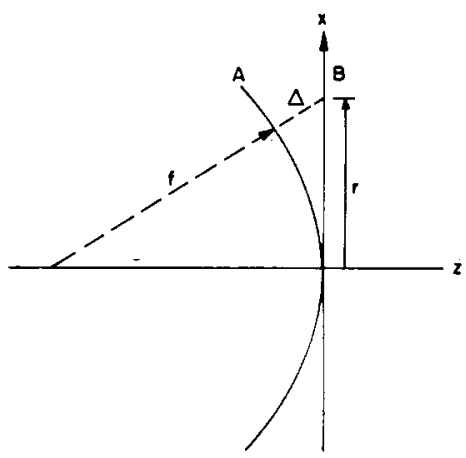

Fig. 14. A lens acts to convert a spherical wavefront (A) into a plane wavefront (b). It must add a phase shift $\exp \left(-j k_{0} \Delta\right)$ where $f^{2}+$ $r^{2}=(f+\Delta)^{2} \approx f+2 \Delta f$ or $\Delta=r^{2} / 2 f=\left(x^{2}+y^{2}\right) / 2 f$.

which either described by a transparency $t(x, y)$ for the transmission mode or a reflectivity $\mathscr{R}(x, y)$ for the transmission mode.

After traversing the object, or being reflected by the object, we can continue this process to again reach the focal plane, transform through the lens, and move from the lens to the transducer to obtain the distribution at plane 6 are denoted by $u_{6}(x, y)$. This is the output, but it is not that parameter that we measure. Rather we measure the transducer voltage $V$. This voltage is simply the integral of the distribution $u_{6}(x, y)$ weighted by the response of the transducer itself at the point $x$ and $y$, i.e., $s(x, y)$ :

$$
V=\iint u_{6}(x, y) s(x, y) d x d y .
$$

It is easy to demonstrate that a unit voltage on the transducer will produce a wave distribution in the crystal, plane 0 , that is just $s(x, y)$. It has also been shown that the principal of reciprocity can be invoked to demonstrate this feature.

\section{B. Beam Contours and Computed Images}

The theory as outlined here can be used to calculate beam contours and spatial frequency content of the converging beam. With these we can compute the images that would be expected for idealized objects. The details of the calculations which do appear in the literature [45] are not appropriate for this review and we will, therefore, proceed directly to the results.

We specify a particular lens at a sapphire-water interface and a frequency of $1 \mathrm{GHz}$. The lens radius is $75 \mu \mathrm{m}$ and the focal length is $83 \mu \mathrm{m}$. The radius of the transducer is $96 \mu \mathrm{m}$ and it is spaced $2 \mathrm{~mm}$ from the lens. The sensitivity of the transducer $s(x, y)$ is assumed to be uniform over the area of the transducer itself. The spatial frequency transfer function of the system produced by this combination is shown in Fig. 15. In Fig. 15(a) we plot amplitude (dotted) and phase (solid) as a function of the spatial frequencies for an ideal lens which neglects all factors such as absorption in the liquid, mode conversion at the lens surface, and a quarter-wave matching layer on this surface. When all of these factors are included the distributions change to that given in Fig. 15(b). The corresponding beam contours at the focal plane are shown in Fig. 16. The expected resolving power for beam contoured as in Fig. $16(\mathrm{~b})$ is $0.7 \lambda_{0}$, i.e., the instrument is capable of resolving a periodic grating with a full period of $0.7 \lambda_{0}$ which is $1.0 \mu \mathrm{m}$ for the wavelength in water is $1.5 \mu \mathrm{m}$ at $1 \mathrm{GHz}$. These numbers are close to the measured values. 


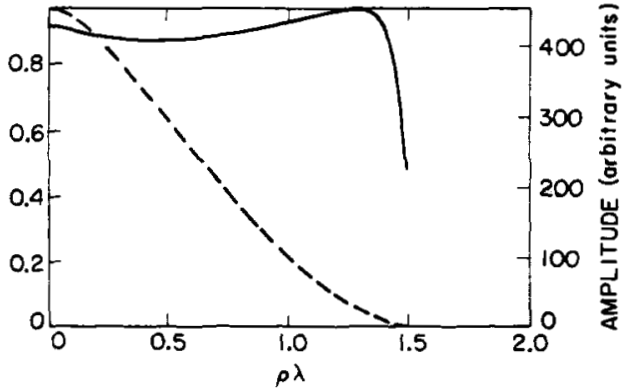

(a)

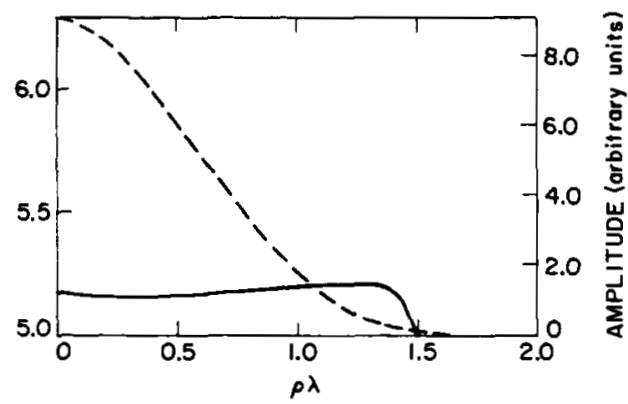

(b)

Fig. 15. The amplitude (dashed) and phase (solid) of the spatial frequency transfer function in terms of the spatial frequency $\rho$ and the wavelength $\lambda$. Curve (a) is for an ideal system. Curve (b) is for a system with spherical aberration, mode conversion, and absorption in the liquid as described in the text.

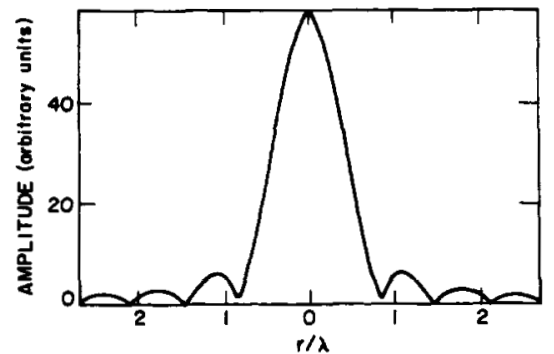

(a)

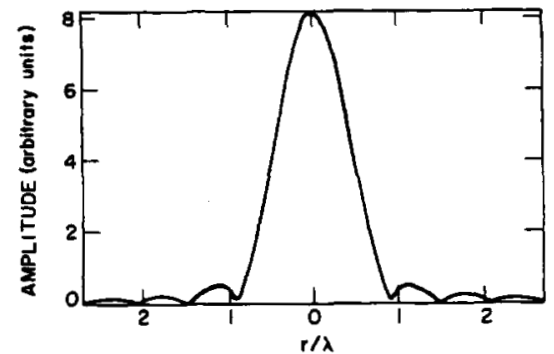

(b)

Fig. 16. The amplitude distribution at the focal plane for the conditions specified in Fig. 15.

The theory can also be used to calculate the expected response of an edge and three different systems are compared in Fig. 17 [28]. There we show a conventional incoherent and coherent optical system together with the coherent scanning system. We see that the confocal scanning system (Fig. 17(c)) compares with the incoherent conventional system. The ringing usually associated with coherent radiation (Fig. 17(a)) is missing. The characterizations as shown in Figs. 16 and 17 are often used but it is necessary to consider the image of an individual object with some care. For example, the circular sidelobes on the beam profile of Fig. 16 do not show up in the

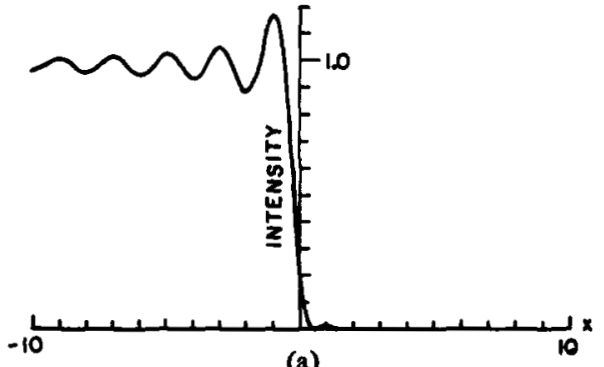

(a)
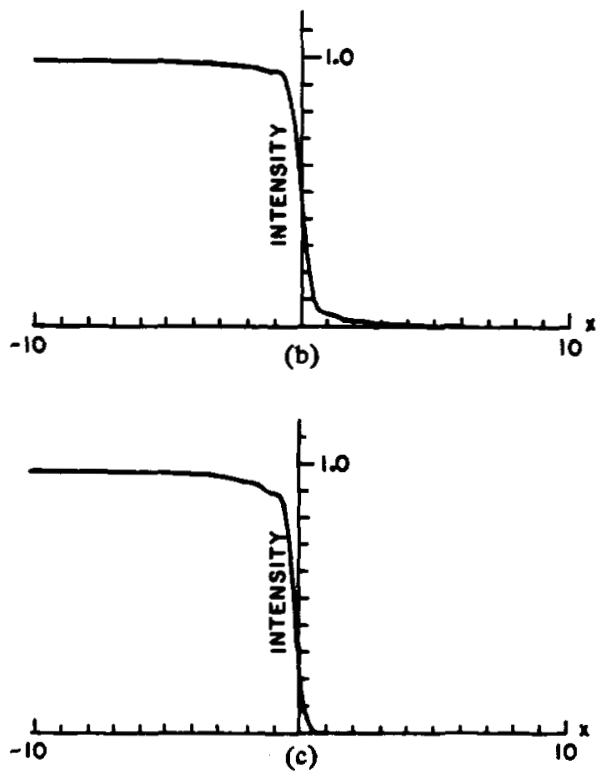

Fig. 17. Calculated one-dimensional images of a step function object. (a) Conventional coherent image. (b) Conventional incoherent image. (c) Image produced by a confocal scanning system using coherent radiation (Lemons [28]).

image of a straightedge as in Fig. 17. They could, however, distort the image of two scattering centers that were closely spaced when their scattering strengths were widely different.

A computed image of the red blood cell is shown in Fig. 18. The object is assumed to be lossless and it only acts to vary the phase of the transmitted beam as shown in Fig. 18(a). The transducer output is plotted in Fig. 18(b) for the phase of the signal and in Fig. 18(c) for the amplitude of the signal. The phase image Fig. 18(b) corresponds quite closely to the object itself. The amplitude image Fig. 18(c) exhibits high contrast even though the amplitude of the scanning beam was assumed to be uniform over the object. The amplitude image of phase objects of this kind does not necessarily conform to the structure of the object. This phenomenon is important for this type of imaging. It can be explained with the aid of the sketch in Fig. 19. We learn from that diagram that a change in object phase over the beam cross section will tilt the transmitted beam. After passing through the output lens the tilted beam will transform into a pseudo-plane propagating off the center of the crystal axis. It will, therefore, be normally incident on the transducer but the transducer response will be diminished as a result of the tilting. The decrease in the magnitude of the output signal is proportional to the gradient of phase in the focal plane. The phase of the transmitted beam is unaffected by this tilt. We do, therefore, expect the phase of the output signal to reproduce the phase of the object with good fidelity. On the other hand, the amplitude of the output signal should 


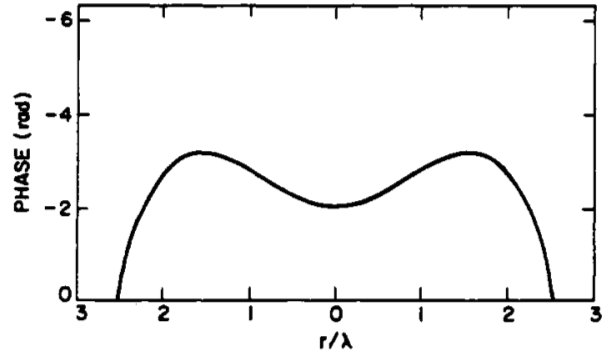

(a)

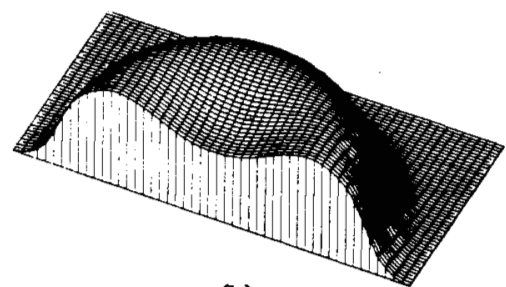

(b)

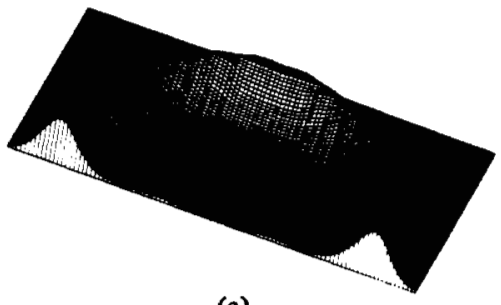

(c)

Fig. 18. Ideal red blood cells. (a) Phase profile from Evans and Fung [46] (hemoglobin absorption equal to water). (b) Phase response of transducer output. Grid lines are $\lambda / 8$ by $\lambda / 16$. (c) Amplitude response of transducer together with the derivative of the phase responses in (b). Grid lines are $\lambda / 16$ by $\lambda / 32$.

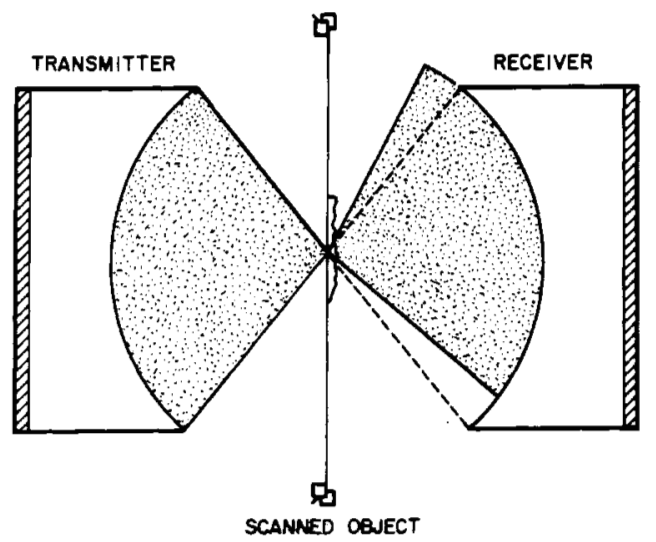

Fig. 19. Illustration of change in illumination of the lens when the beam encounters a transverse phase gradient in the focal plane.

correspond to the phase gradients in the object plane. We see both of these features in Fig. 18(b) and (c).

These ideal properties are changed if the focal conditions are not precise. Defocusing can be of two types-either a) the object is not placed at the focal plane, or b) the spacing between the lens in Fig. 13 does not equal to confocal conditions. These two defocusing conditions are illustrated as follows. In Fig. 20 we show the spatial frequency content of the beam for (a) the in-focus condition, (b) the object defocused from the focal plane by one wavelength, and (c) the lens separation is decreased from the confocal condition by one wavelength. There are changes in both the phase and amplitude of the spatial frequencies, and they alter the images as shown in Fig. 21. The translation of the object from the focal plane

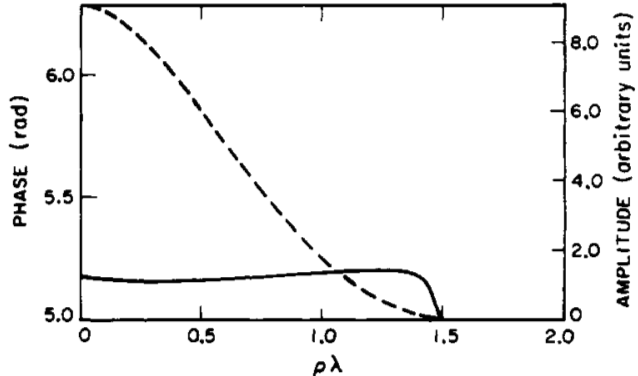

(a)

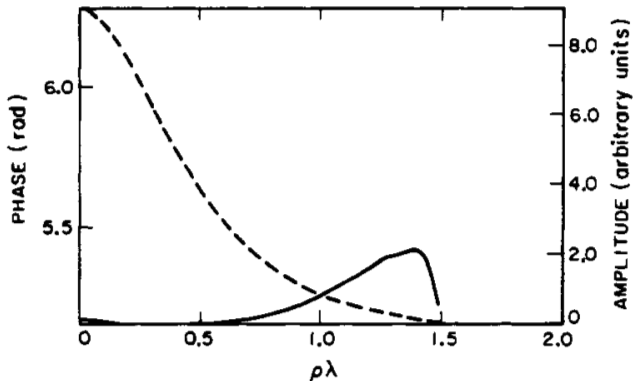

(b)

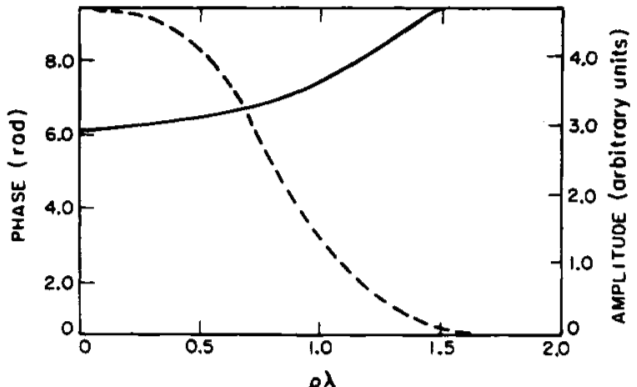

(c)

Fig. 20. Spatial frequency transfer function amplitude (dashed line) and phase (solid line) for various cases. (a) Lenses are confocal and the object is at focus. (b) Lenses are confocal and the object is defocused by one wavelength. (c) Transmitter-receiver lens spacing decreased by one wavelength.

makes a small difference in the contrast and broadens the image somewhat. But the more dramatic distinction comes from the images in Fig. 21(c), where the lens spacing is decreased by one wavelength. We see there in the amplitude image a contrast reversal which is different in character from the image. This type of reversal for focusing errors in optical systems has been discussed by Goodman [47].

Finally, in Fig. 22 we present the red blood cell images as they appear when the focusing errors of Fig. 21 are introduced. The most pronounced feature in this series is again the contrast reversal in the amplitude images when the lens spacing decreases (Fig. 22).

\section{The $V(Z)$ Curves}

For the reflection instrument the output voltage as expressed in (5-14) can be used in a unique way to measure some of the elastic properties of the reflecting surface. The initial distribution at the transducer is $u_{0}^{+}(x, y)$, the distribution of the returning field is $u_{0}^{-}(x, y)$, and we have

$$
V(Z)=\int_{-\infty}^{+\infty} \int_{0}^{+}\left(x^{\prime}, y^{\prime}\right) u_{0}^{-}\left(x^{\prime}, y^{\prime}\right) d x^{\prime} d y^{\prime}
$$

In writing this form we are anticipating the final result wherein we monitor $V$ as a function of the parameter $Z$; i.e., the dis- 


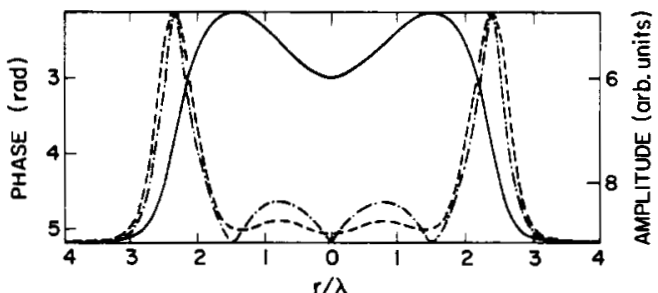

(a)

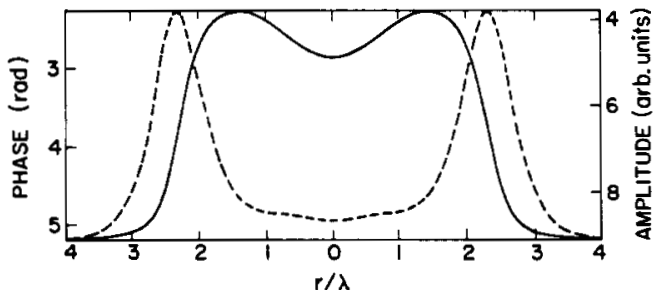

(b)

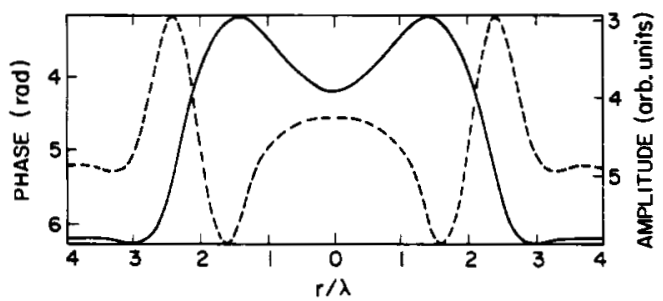

(c)

Fig. 21. Computed amplitude (dashed line) and phase (solid line) image of the ideal red blood cell with the conditions specified in Fig. 20.

placement of the reflecting object from the focal plane. Equation (5-14) can take on a more suitable form by using the transforms described previously. The details are published elsewhere [48]. The final form for $V(Z)$ is as follows:

$$
\begin{aligned}
& V(Z)=\int_{-\infty}^{+\infty} \int_{1}\left(u_{1}^{+}(x, y)\right)^{2}(P(x, y))^{2} \\
& \quad \times \Re\left(\frac{x}{f}, \frac{y}{f}\right) \exp \left(-j \frac{k_{0} Z}{f^{2}}\left(x^{2}+y^{2}\right)\right) d x d y .
\end{aligned}
$$

Since our problem exhibits circular symmetry about the $z$ axis we can replace $x^{2}+y^{2}$ by $r^{2}$ and write [48]

$$
V(Z)=\int_{0}^{\infty} r\left(u_{1}^{+}(r)\right)^{2}(P(r))^{2} \Re\left(\frac{r}{f}\right) \exp \left(-j \frac{k_{0} Z}{f^{2}} r^{2}\right) d r .
$$

An alternative form of this expression and its derivation may be found in the literature [49]. This $V(Z)$ expression has proved to be valuable as a method for studying surfaces. Some results for real cases will be given in a later section. Here we want to gain some understanding of what is to come by analyzing a few simple reflectors.

One straightforward case is that of a lens with a uniform illumination, i.e., $u_{1}(r)=1$, and radius $R$, i.e., $P(r)=\operatorname{circ}(r / R)$ and a perfect reflector $R(r / f)=1$. In this case (5-15) becomes

$$
V(Z)=\int_{0}^{R} r \exp \left(-j \frac{k_{0} Z}{f^{2}} r^{2}\right) d r=\frac{R^{2}}{2} \exp (-j x) \operatorname{sinc} x
$$
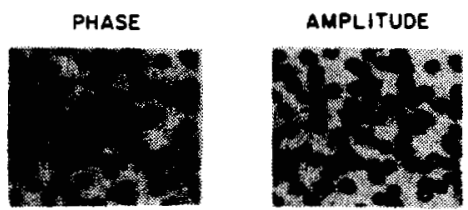

(a)
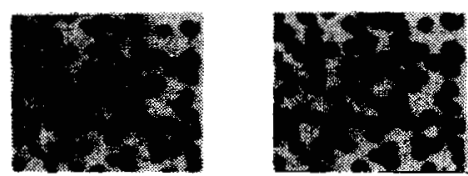

(b)
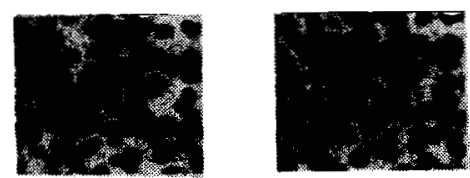

(c)

Fig. 22. Experimental images of red blood cells for the three conditions specified in Fig. 20. Note the reversal of contrast in amplitude for (c) the favorable comparison with the theory in Fig. 21(c).

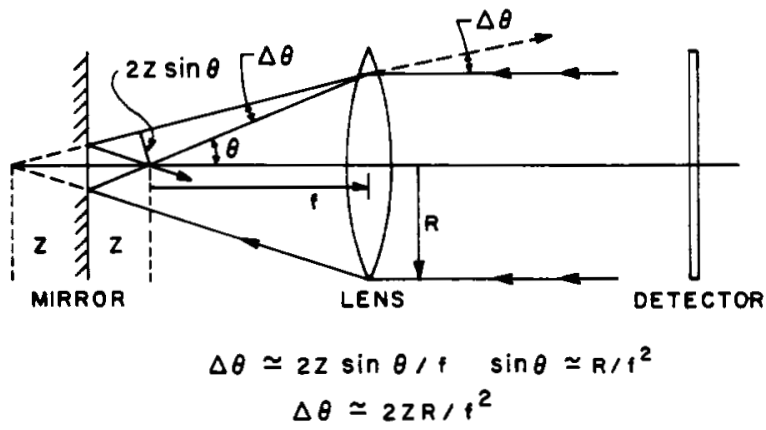

(a)

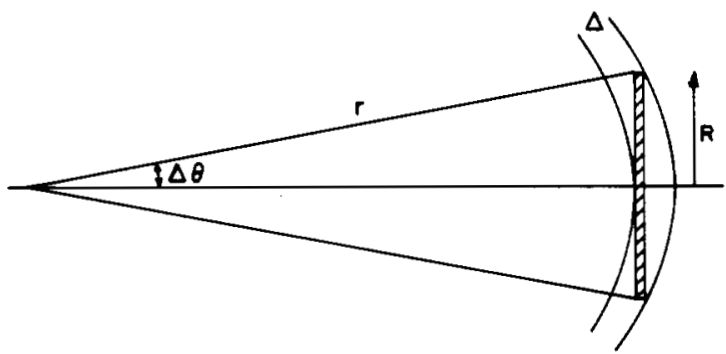

$$
\begin{aligned}
& r=R / \Delta \theta \simeq f^{2} / 2 z \text { (from above) } \\
& r^{2}+R^{2}=(r+\Delta)^{2} \simeq r^{2}+2 \Delta r \\
& \Delta=R^{2} / 2 r=\left(R^{2} / f^{2}\right) z
\end{aligned}
$$

(b)

Fig. 23. Sketch of rays for a displaced mirror. (a) Reflection from mirror displaced by $z$ from the focal plane. (b) Phase variation across transducer for a spherically diverging wave.

where

$$
x \equiv \pi(R / f)^{2} Z / \lambda_{0} \text { and } \operatorname{sinc} x \equiv \sin x / x .
$$

The result in (5-16) is easily understood. When $Z$ is positive the wavefront that impinges on the transducer is spherical with a radius of curvature that depends on $Z$. As $Z$ is changed we experience an increasing number of cyclic variations of the field across the transducer which gives rise to the sinc curve.

It is instructive to use this concept and calculate the value of $Z$ for the first null in the transducer output. The sketch in Fig. 23 is sufficient for this purpose. We can see from Fig. 


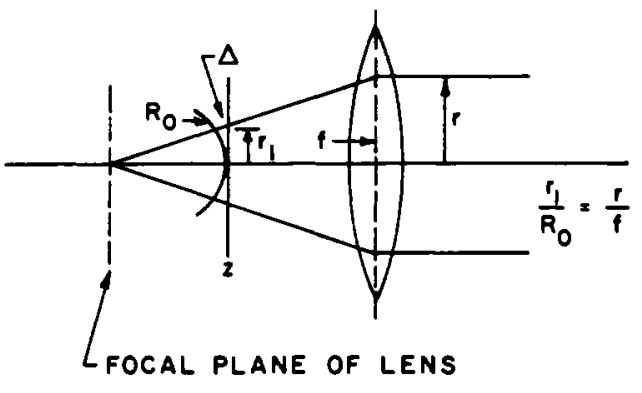

$$
\begin{aligned}
& R_{0}+r_{1}^{2}=\left(R_{0}+\Delta\right)^{2} \simeq R_{0}^{2}+2 \Delta R_{0} \\
& 2 \Delta=r_{1}^{2} / R_{0}=R_{0} r^{2} / f^{2}
\end{aligned}
$$

Fig. 24. Illustration of phase shifts associated with a spherical dome of radius $R_{0}$ centered on focal plane.

23(b) that the response of the transducer will be reduced to zero when the wave at the outer rim undergoes an additional phase shift of $2 \pi$ as compared to the portion of the wave at the center of the transducer $\left(\Delta=\lambda_{0}\right)$. We see from Fig. 23(a) that this occurs when the radius of curvature of the converging wave is adjusted to be $R^{2} / 2 \lambda_{0}$. From (b) we find that a displacement of the reflector from the focal plane by an amount $Z$ will produce a radius of curvature in the converging wave given by $r=f^{2} / 2 Z$. In turn this is equal to $R^{2} / 2 \lambda_{0}$. Therefore, we have the expression $Z=\lambda_{0}\left(R^{2} / f^{2}\right)$ for the null condition at the transducer. This agrees with $(5-16)$ when we note that the sinc function has a null for $x=\pi$ (and $Z / \lambda_{0}=(R / f)^{2}$ ).

In the second example as illustrated in Fig. 24 the reflecting object is a dome with a radius of $R_{0}$ and centered on the focal plane. We see from the sketch that on the plane spaced a distance $R_{0}$ from the focal plane, the waves suffer an additional phase shift of $k_{0}(2 \Delta)$ where $\Delta$ is given by

$$
r_{1}^{2} / 2 R_{0}=\frac{R_{0}}{2 f^{2}} r^{2} .
$$

The factor of $2 \Delta$ is entered since the wave going and returning traverses this distance twice. We are at liberty to represent this dome by a surface with a reflectivity given by

$$
\Re=\exp \left[-j k_{0}\left(\frac{R_{0}}{f^{2}}\right) r^{2}\right]
$$

and in place of (5-16) we have

$$
\begin{aligned}
V(Z) & =\int_{0}^{R} r \exp \left[-j k_{0}\left(\frac{R_{0}}{f^{2}}\right) r^{2}\right] d r \\
& =\frac{R^{2}}{2} \exp \left(-j x_{2}\right) \operatorname{sinc} x_{2}
\end{aligned}
$$

where

$$
x_{2}=\pi(R / f)^{2}\left(Z+R_{0}\right) / \lambda_{0} .
$$

This result is similar to (5-16) except that the reflector must be located at $Z=-R_{0}$ to give the maximum output. This is intuitively correct since at that point the curvature of the dome equals the curvature of the wavefront.

For the third case we consider the special situation where the acoustic impedance of the liquid is equal to that of the solid. However, the velocity ratio is still small and the critical

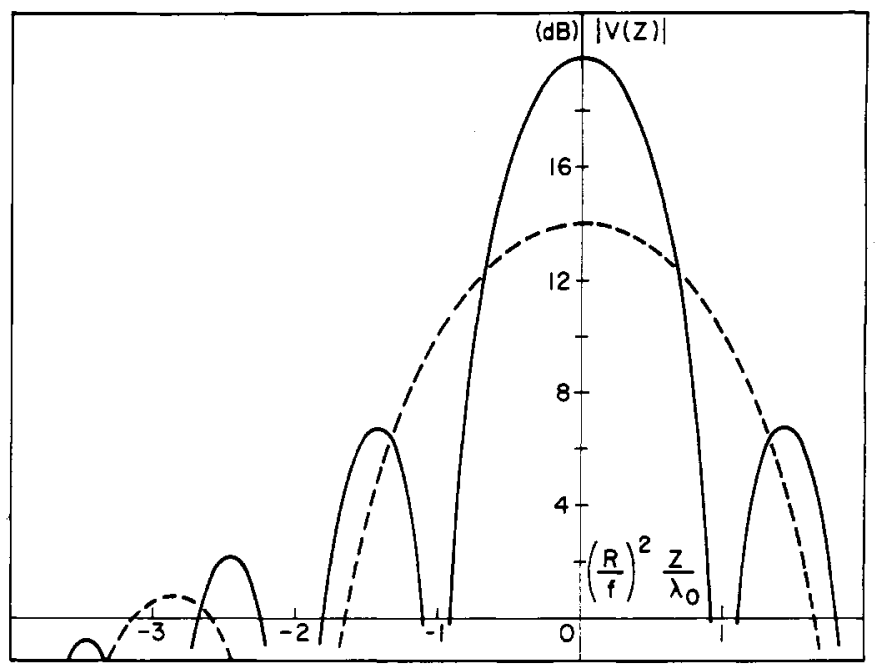

Fig. 25. $V(Z)$ curves of a uniformly illuminated lens for a perfect reflector (solid line) and for a reflector matched to the liquid up to a critical angle (dashed line).

angle for total internal reflection occurs within the convergence of our acoustic beam. (The interface between mercury and silicon approximates this condition.) The reflectance becomes

$$
\Re=\left\{\begin{array}{lc}
0, & r<R_{1} \\
1, & R_{1}<r<R
\end{array} .\right.
$$

For this case we can still use (5-17) except that the limits on the integration now extend from $R_{1}$ to $R$. The result is

$$
V(Z)=\frac{R^{2}-R_{1}^{2}}{2} \operatorname{sinc} \frac{k_{0}}{2} \frac{R^{2}-R_{1}^{2}}{f^{2}} .
$$

It is similar to the first case but the null now occurs at $Z \lambda_{0}=$ $f^{2} /\left(R^{2}-R_{1}^{2}\right)$ which is larger than that of the perfect reflector. A comparison of (5-18) with (5-16) is shown in Fig. 25 for the case where the area of transducer within $R_{1}$ is equal to the area between $R_{1}$ at $R$, i.e., $R=\sqrt{2} R_{1}$.

For the fourth case we choose a reflector that more closely follows the characteristics of the actual liquid-solid interface encountered with real objects. The reflectivity for this case is of the form

$$
\mathfrak{R}= \begin{cases}1, & r<R_{1} \\ \exp (j \phi), & R<r<R_{1} .\end{cases}
$$

With this we can write

$$
\begin{aligned}
V(Z)=\int_{0}^{R_{1}} r \exp \left[-j\left(\frac{R_{0}}{f^{2}}\right) r^{2}\right] d r & \\
& \quad+\exp (j \phi) \int_{R_{1}}^{R} \exp \left[-j\left(\frac{R_{0}}{f^{2}}\right) r^{2}\right] d r .
\end{aligned}
$$

This when evaluated reads

$$
\begin{array}{r}
V(Z)=\frac{R_{1}^{2}}{2} \operatorname{sinc} \frac{k_{0}}{2}\left(\frac{R_{1}}{f}\right)^{2} Z+\frac{R^{2}-R_{1}^{2}}{2} \exp \left[-j\left(\frac{k_{0}}{2}\right)\left(\frac{R}{f}\right)^{2} Z\right] \\
\times \exp (+j \phi) \operatorname{sinc} \frac{k_{0}}{2} \frac{\left(R^{2}-R_{1}^{2}\right)}{f^{2}} Z
\end{array}
$$




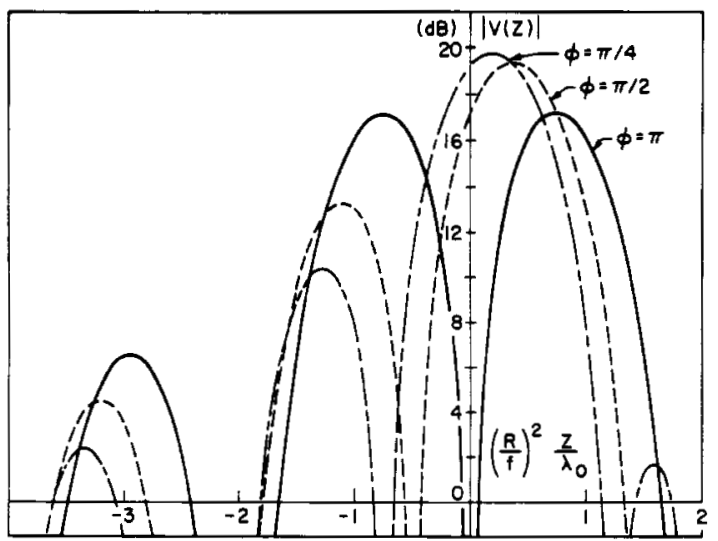

Fig. 26. $\overline{V(Z)}$ curve of a uniformly illuminated lens for a reflector with a unit amplitude reflectance function. Reflectance function phase is nonzero for angles exceeding a critical value. Different $V(Z)$ curves are for various phase values.

The curves resulting from different values of $\phi$ are shown in Fig. 26.

These simple cases tell us a great deal about the character we expect to find in the measured $V(Z)$ curves. A reduction of the reflectivity for incident angles less than the critical angle will tend to broaden the curve and a phase shift for angles greater than the critical angle will tend to displace the curves.

There is one other important feature that comes forthnamely contrast reversal in the images as $Z$ is varied [15], [50]. Assume that we have an image composed of two materials-one with the reflectivity as in the second case, and the other as in the perfect reflector. For $Z=0$ the perfect reflector would have the larger reflectivity and would appear brighter in the image (Fig. 25). For negative values of $Z$ we see that the contrast is reversed particularly at the null where the ideal reflector goes dark and the reflector with the matched liquid remains fairly bright.

The most important feature that has been left out of these examples is the nonuniform illumination of the lens. This can arise from several factors: diffraction over the length of the crystal, a nonuniform response of the transducer over its cross section, the axis of the transducer displaced from the lens axis through misalignment in the initial fabrication, or the quarterwave matching layer can exhibit inhomogeneities. For all of these reasons, and more, we find actual distributions to differ from the ideal that has been postulated in the previous examples. The most pronounced effect in the $V(Z)$ curves is the disappearance of the nulls and their replacement with dips of varying degrees of depth.

\section{Reflectance Functions}

We now attend to the problem of calculating the reflectance of plane waves on a liquid-solid interface as a function of the angle of incidence. The problem is straightforward and it has been treated in the literature [51]. Our needs are somewhat peculiar in that we must cover the entire range of incident angles. The operating frequency is high enough to make the attenuation of the liquid important but for the most part we deal with a linear problem in classical wave theory. The problem is sketched in Fig. 27. We express the reflected wave in terms of the incident wave by the reflection coefficient $R$. We require both the magnitude and the phase of $R$ as the incident angle $\theta$ (or more properly $\sin \theta$ ) is varied. In the solid we find that several modes can propagate which involve longitudi-

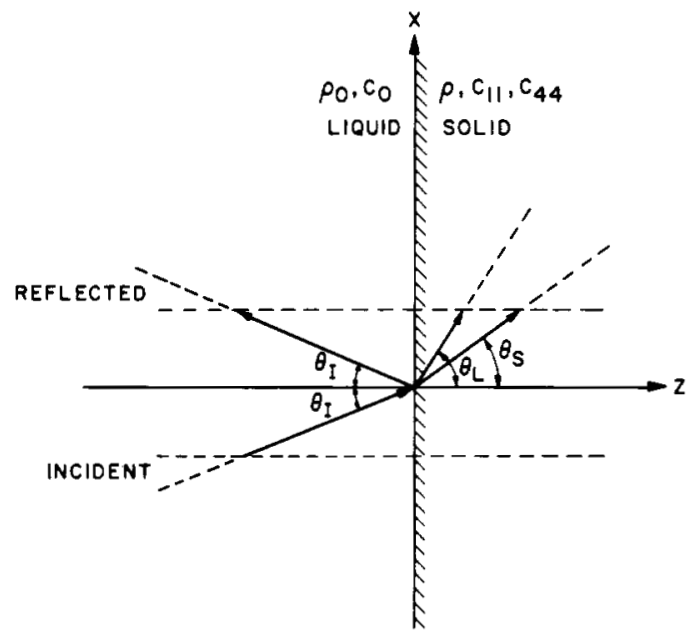

Fig. 27. Acoustic plane wave scattering at a plane boundary between a liquid and an isotropic solid.

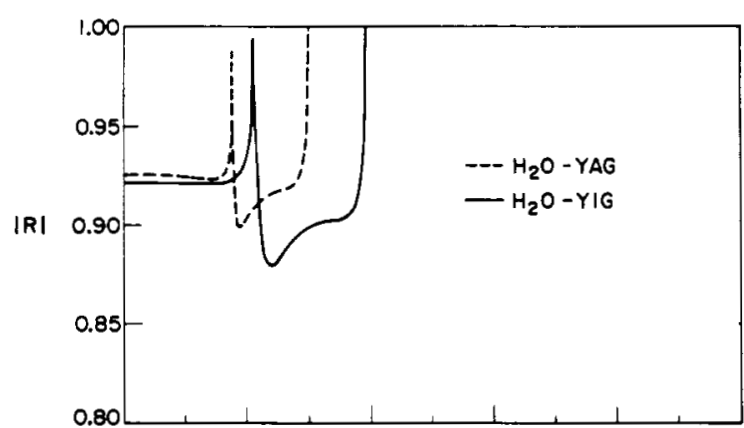

(a)

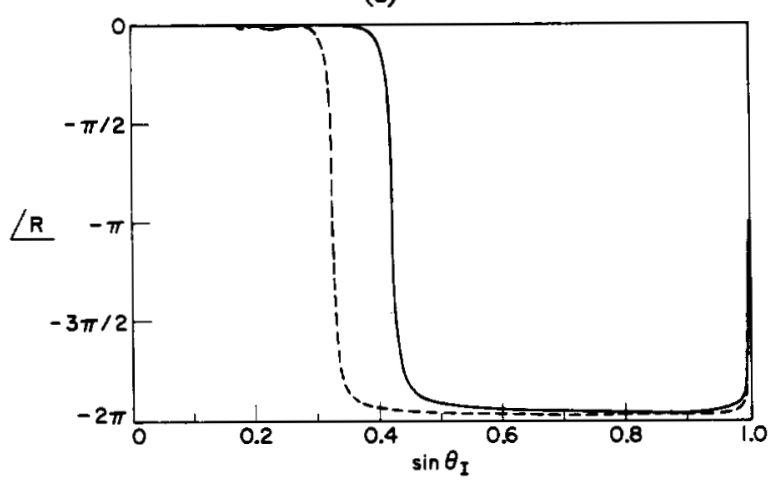

(b)

Fig. 28. Amplitude and phase of the reflectance function for $\mathrm{H}_{2} \mathrm{O}-\mathrm{Y} 1 \mathrm{G}$ and $\mathrm{H}_{2} \mathrm{O}-\mathrm{YAG}$ interfaces.

nal, shear, and Rayleigh waves along the interface. In layered media other modes representing energy propagating within the layer will enter.

For this review it is only required that we present the final form of the reflectance functions since it is this parameter that enters into the expression that we use to calculate the $V(Z)$ curves. A typical result is given in Fig. 28 for two different crystals (YAG and YIG) against water. The magnitude of $R$ is rather uninteresting since the results would not be altered appreciably if we set $R=1$ over the entire range. But the phase $R$ is crucial. We see that the phase of the reflected wave undergoes a large shift at the critical angle and approaches $2 \pi$ for large angles. The range near the critical angle is important where the phase relation between the reflected wave and the incident wave undergoes a maximum of change. The boundary conditions that determine this condition require continu- 

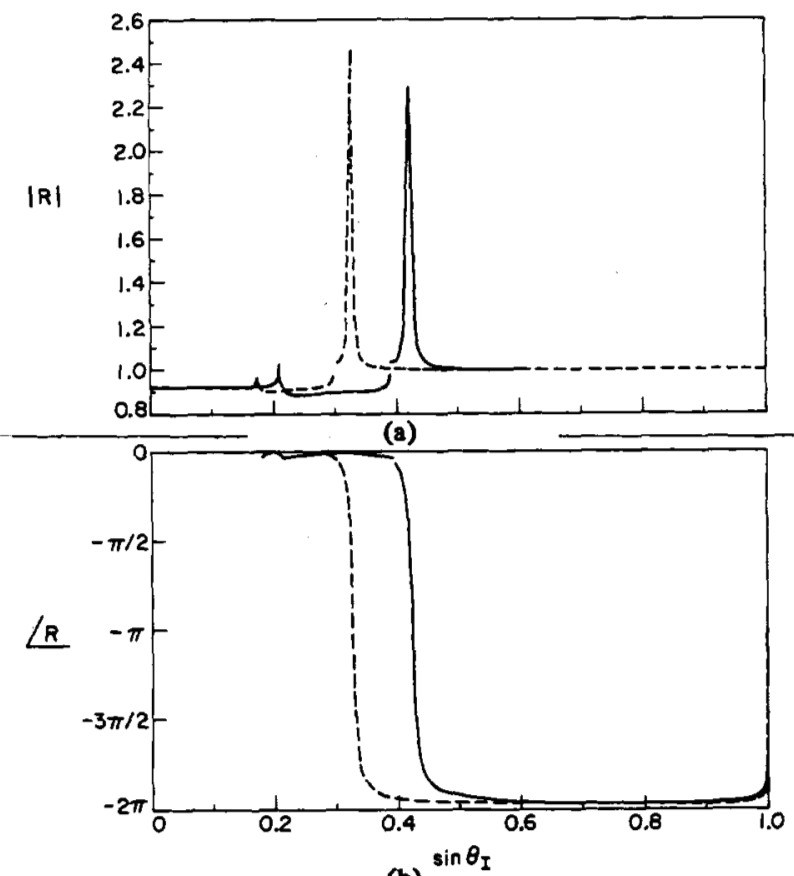

(b)

Fig. 29. Amplitude and phase of the reflectance function for lossybiquid-lossless-solid interface. Solid line $\mathrm{H}_{2} \mathrm{O}$-YIG interface, dashed line $\mathrm{H}_{2} \mathrm{O}-\mathrm{YAG}$ interface $\left.\left(\alpha V_{0} / \omega\right)=5.09 \times 10^{-3}\right)$.

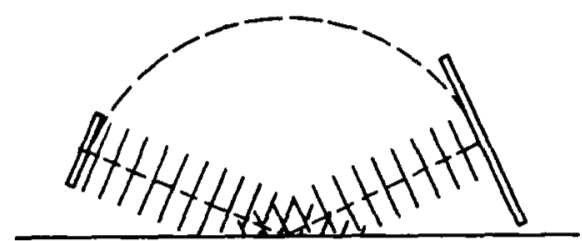

(a)

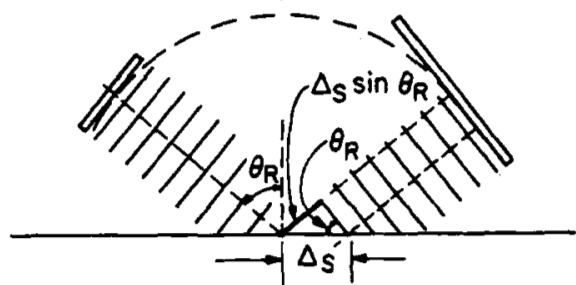

(b)

Fig. 30. Mustration of a setup to measure the reflectance function. (a) At an angle larger than the Rayleigh angle. (b) At the Rayleigh angle. In (b) liquid path is less than (a) by an amount $\Delta_{S} \sin \theta_{R}$.

ity in both the stress and particle velocity across the interface. If the phase of the reflected wave for normal incidence is equal to the incident wave, it must differ from that value at the critical angle. This follows from the fact that a large component of the Rayleigh interface is excited near the critical angle. When the components of this wave are put into the boundary conditions the phase of the reflected wave must be adjusted to compensate for this addition and the variation of Fig. 28 reflects this adjustment.

As previously mentioned, the acoustic loss of the liquid is important at our frequencies. When this factor is included in the theory, the curves of Fig. 28 are modified and one interesting feature comes in-namely, the magnitude of $\Omega$ can exceed unity near the critical angle as shown in Fig. 29 [52]. In the literature we find this curve has a dip at this angle for a lossless liquid interfacing with a lossy solid. There the dip is explained by the fact that the Rayleigh wave excited at this
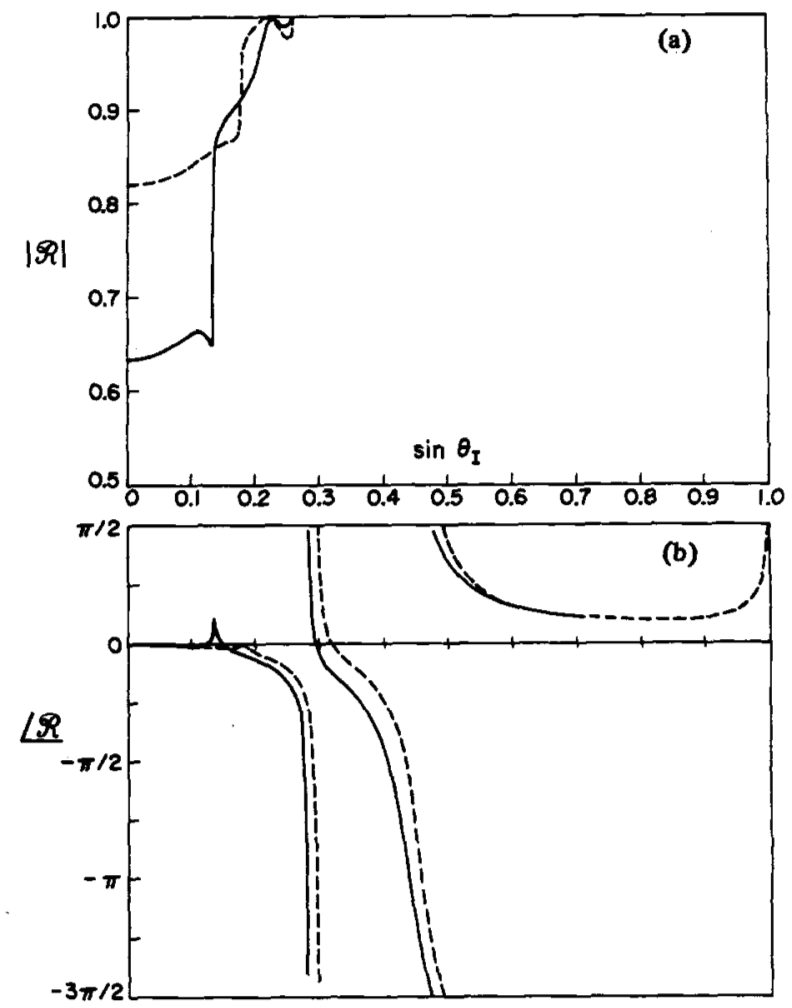

Fig. 31. Reflectance function amplitude (a) and phase (b) for a $\lambda / 4$ thick aluminum layer on sapphire (solid line) and silicon (dashed line) substrates.
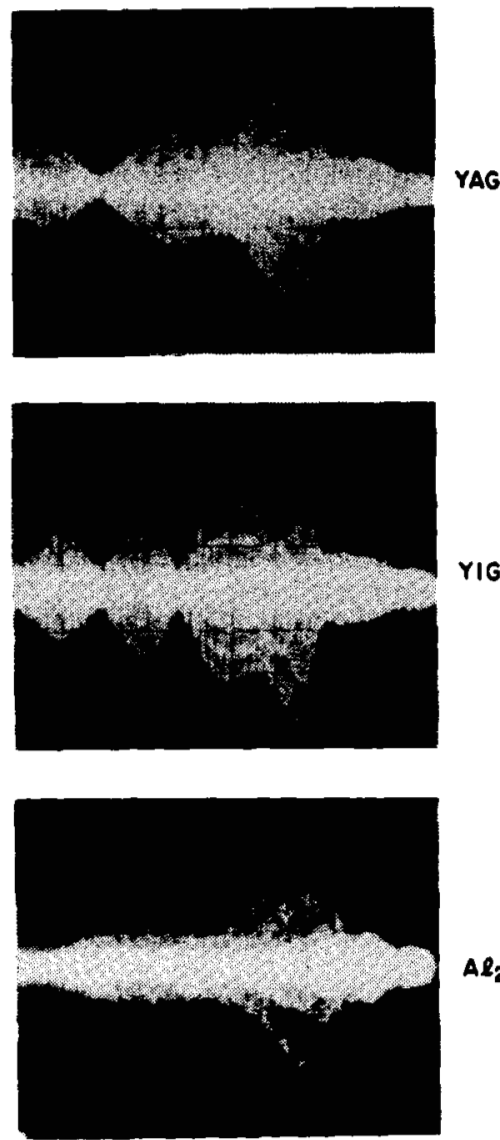

$\mathrm{Al}_{2} \mathrm{O}_{3}$

Fig. 32. Long exposure oscilloscope photos of the returning pulses as the distance between the sample and lens is varied. Envelopes show $V(Z)$ curves for different crystals. Horizontal scale $3.75 \mu \mathrm{m}$ / $\operatorname{div}(1100 \mathrm{MHz})$. 


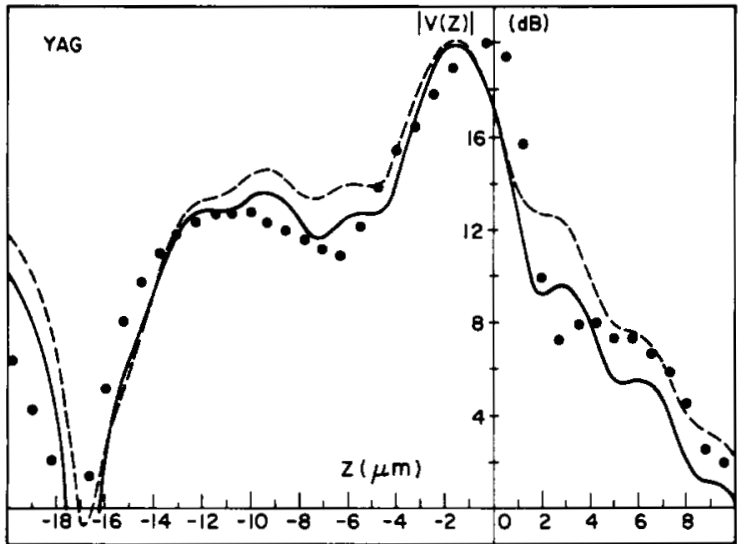

(a)

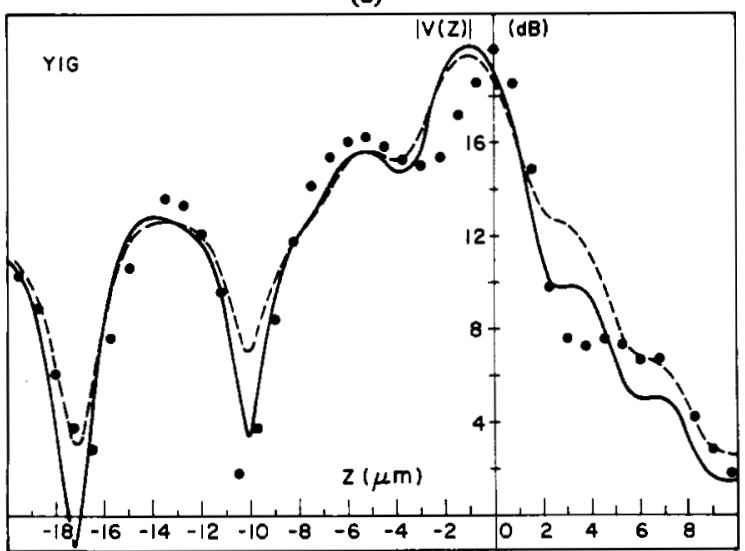

(b)

Fig. 33. Experimental (dots) and calculated (dashed lines) $V(Z)$ curves for YAG and YIG crystals at $1100 \mathrm{MHz}$. Solid lines show the calculated $V(Z)$ curves if an offset of $20 \mu \mathrm{m}$ is assumed between the axes of the lens and transducer.

angle displaces the reflected beam from the position it would have had in a mirror-like reflection-the Schoch displacement [51]. During this lateral displacement the energy is predominately carried by the Rayleigh wave in the solid. The loss associated with propagation in this medium dissipates energy and reduces the energy in the reflected component. For our case with a lossy fluid and a lossless media the situation is reversed. When the reflected beam is displaced along the boundary via excitation of the Rayleigh wave the total path in the lossy liquid is less than it would be for a mirror-like reflection. This point is clarified by the geometry as sketched in Fig. 30. Since the reflection coefficient for the perfect mirror-like reflector is defined as unity, the increased amplitude that appears with the lateral displacement must be accounted for by using a value of $\mathfrak{R}$ greater than unity.

Finally we can illustrate the reflectance for a layered medium. In Fig. 31 we show the results for a layer of Aluminum on two substrates-silicon and sapphire. The additional transitions that are found in these curves are due to the various modes such as Love waves and Sezawa waves that are excited in the presence of the layers.

With these curves at hand we can use the theory as developed in the previous section to predict the $V(Z)$ curves. The measured values of $V(Z)$ for single crystals of YAG, YIG, and $\mathrm{Al}_{2} \mathrm{O}_{3}$ are shown in Fig. 32, and the comparison with the theory is shown in Fig. 33. The effect of layering is shown in Fig. 34, where we have two different thicknesses of Aluminum on Silicon substrates together with some calculations of layering in Fig. 35.
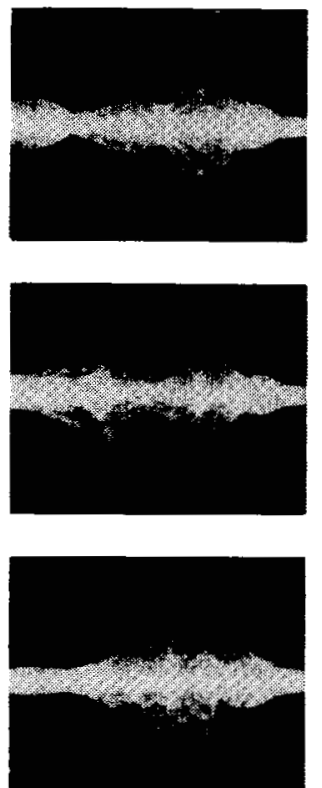

Fig. 34. $V(Z)$ photographs for silicon with different thicknesses of aluminum deposited on it.

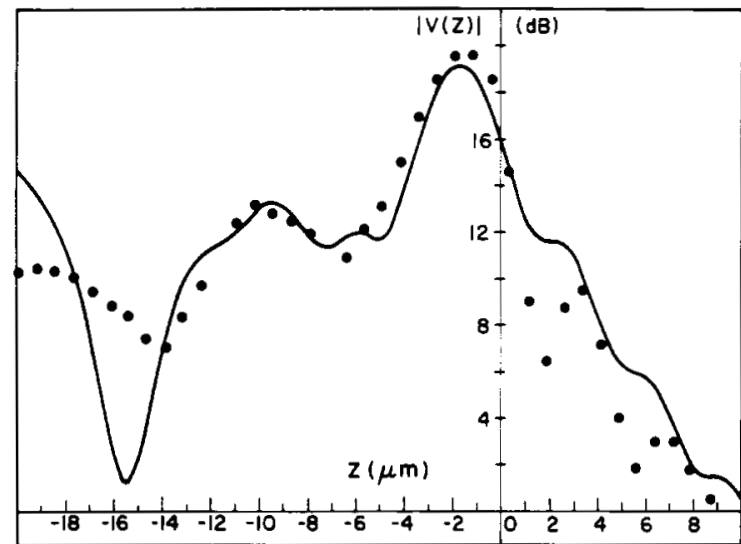

(a)

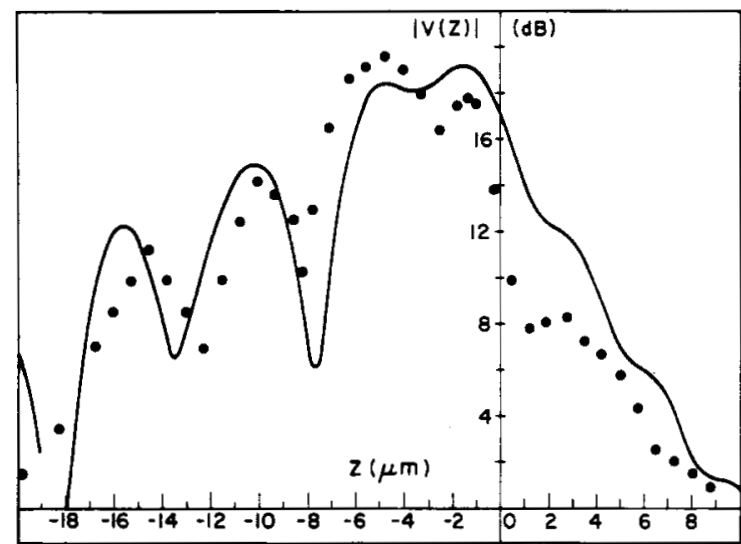

(b)

Fig. 35. Experimental (dots) and calculated (solid lines) $\bar{V}(Z)$ curves for $0.5 \mu \mathrm{m}$ (a) and $2.0 \mu \mathrm{m}$ (b) aluminum layer on silicon substrate (1100 MHz).

Most crystals exhibit anisotropy in their elastic properties and this has an important bearing on the measured response. In Fig. 36 we show the change in the computed curve when anisotropy is included. The change allows for an improved comparison with the experimental points. 


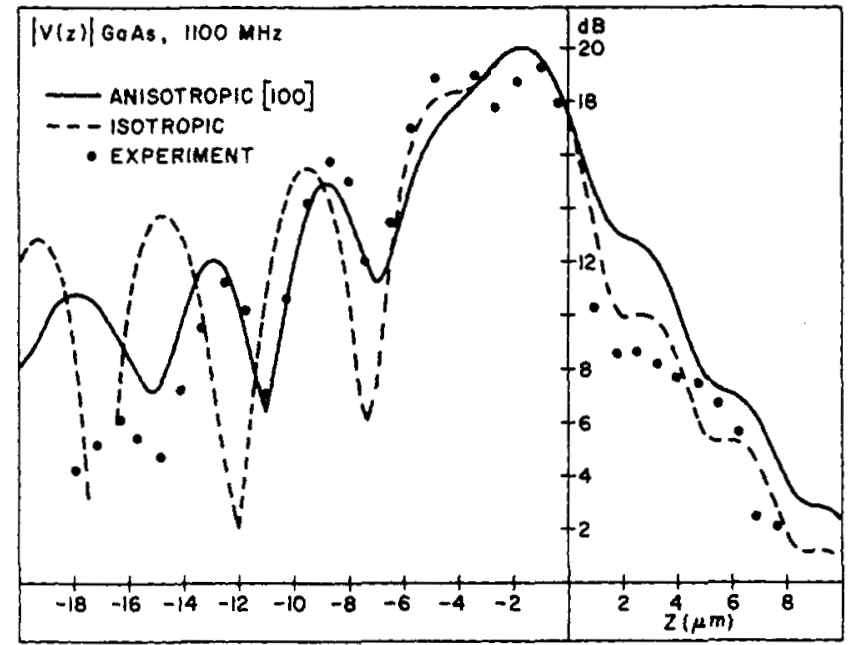

Fig. 36. Experimental (dots) and calculated (solid line) $V(Z)$ curves for GaAs. $V(Z)$ curve resulting from an isotropic calculation (dashed line) is also shown.

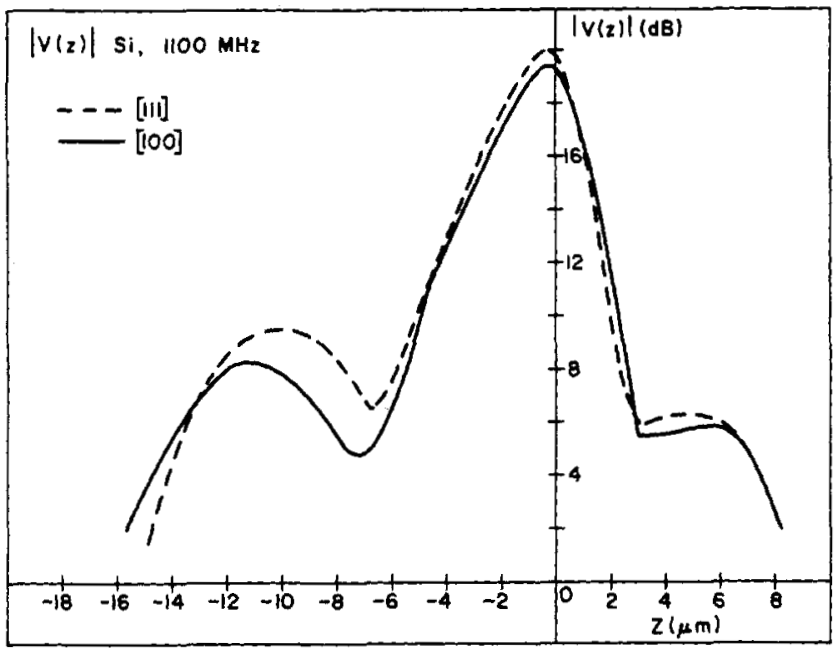

Fig. 37. Measured $V(Z)$ curves for $(100)$ and (111) faces of silicon.

As a final example of what can be done with these curves we show the results of Fig. 37. We see from these that it is possible using this technique to distinguish the [111] reflecting face from the [100] reflecting face. We should not be surprised to see variations in reflectivity as a "source of contrast" in polysilicon or other alloys which are made up of grams of various orientations.

\section{The Images}

The end game for a microscope of any form is the images themselves, for they represent the cumulation of all separate components in the instrument. We have selected representative images as divided into the areas of 1) biological specimen, 2) materials, and 3) integrated circuits. Each of these areas will require specially adapted modifications in final versions and the theory for contrast will differ in the three categories. The biological specimen come from cells of the blood and from a tissue section from the human eye. In Fig. 38 we present a comparison of the optical and acoustic micrographs for the human red blood cell. The cells in the optical image are stained and placed under a cover slip. The cells in the acoustic micrograph are unstained but fixed in methanol. We can see that these images in reflection are of good fidelity at this frequency.

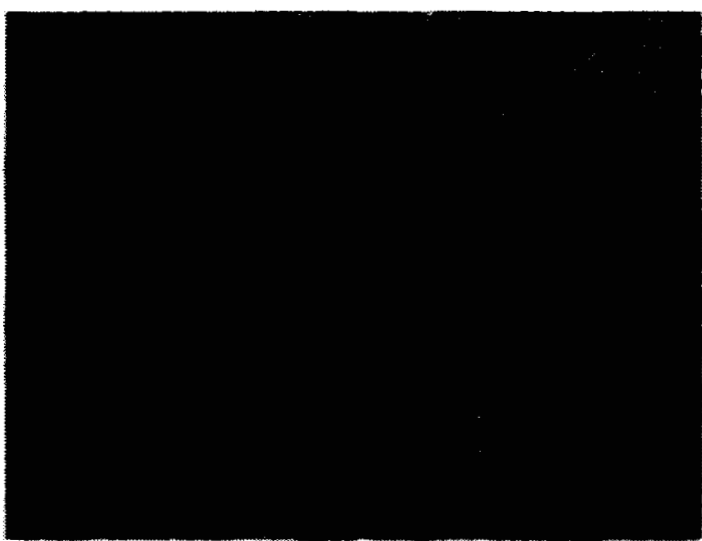

(a)

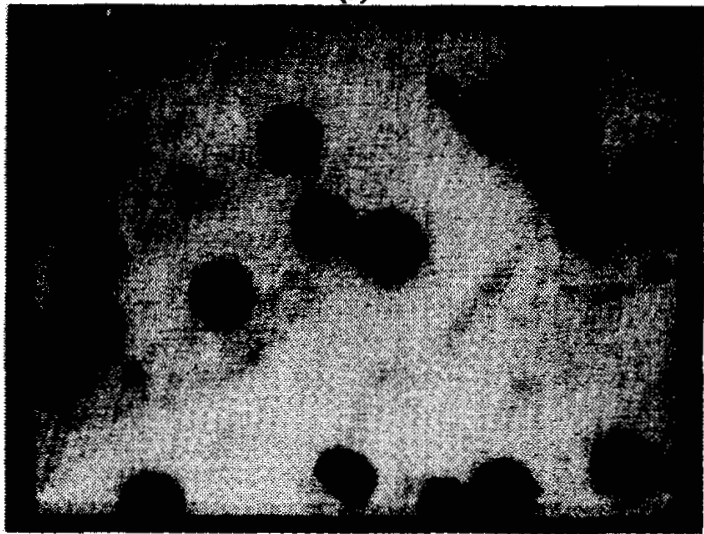

(b)

Fig. 38. Normal red blood cells, (a) Optical fixed in methanol with Leishman's stain (X1000 (oil immersion)). (b) Acoustic (1100 MHz) fixed in methanol, unstained, but mounted on silicon wafer with 1.56 $\mathrm{\mu m}$ of $\mathrm{SiO}_{2}(\times 1000)$. Cells are from different individuals. (Courtesy of $\mathrm{J}$. Heiserman.)

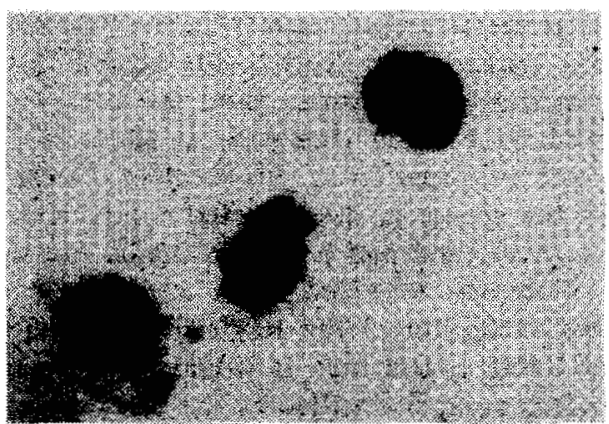

(a)

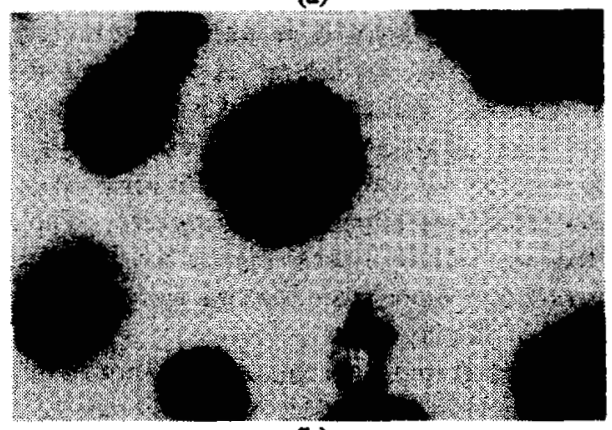

(b)

Fig. 39. Acoustic images in reflection (1100 MHz)-Living macrophage cells.

In Fig. 39(a) and (b), we show the appearance of living white cells (macrophage). These are, of course, unstained, and the two different sets of cells display excellent contrast between 


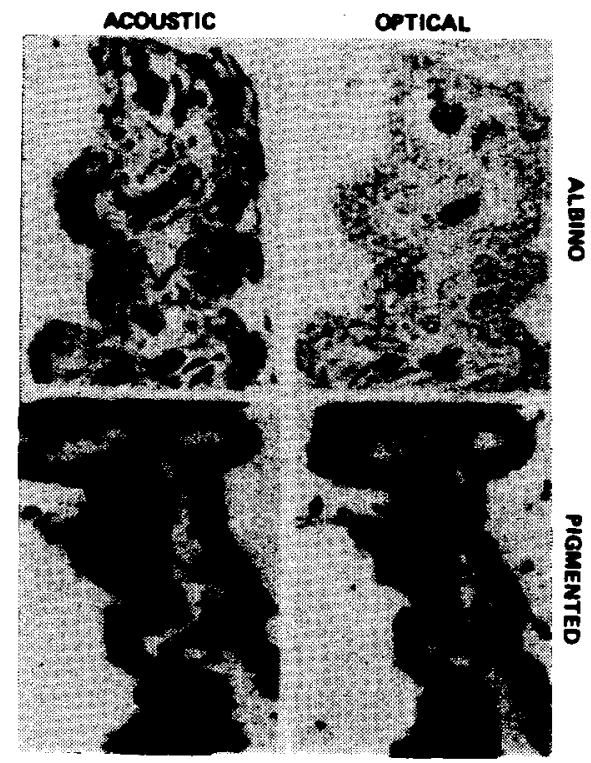

Fig. 40. Sections of iris from an albino and pigmented rabbit (Marmor [53]).
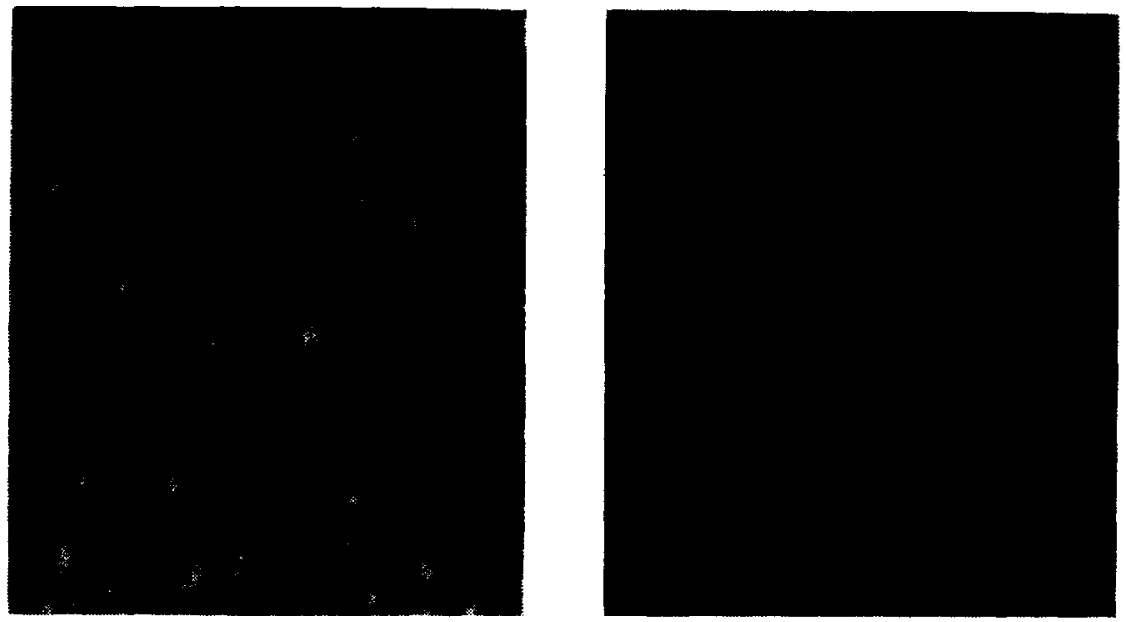

(a)

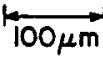

(b)

Fig. 41. Comparison of optical and acoustic images of a Co-Ti alloy. (a) Optical D/C. (b) Acoustic. Co-Ti alloy showing 4 phases. Overall composition 59-percent Co, 41-percent Ti.

the nucleus and the surrounding cytoplasm. It suggests that this instrument may be suitable for studying objects of this sort in the living state. And to complete the set we compare the optical and the acoustic images of tissue sections as taken from the retina of a rabbit in Fig. 40 [53]. Again the acoustic image shows the important parts with a contrast that is slightly improved over that of the optical. These images were taken in transmission.

The next sequence of images are included to demonstrate the acoustic response of materials of various kinds. Perhaps the most spectacular is that shown in Fig. 41-a polished surface of a cobalt-titanium alloy. The optical image on the left shows up the hillocks that result from the polishing technique. There is a slight hint of structure in this image but for the most part the optical reflectivity is almost constant over the surface. The acoustic micrograph is quite distinct. There are actually four levels in this image-two in the light grey regions and two in the darkened regions. We have analyzed these particular areas with an $\mathrm{X}$-ray microprobe and find $a$ one-to-one correspondence between the distribution of phases in this material and the acoustic reflectivity. Stated in a different way, one can determine from the phase diagram for this particular alloy that four different phases-each with a different percentage of titanium-are possible. Each of the four phases has a distinct elastic constant, and it therefore follows from the discussions in Section $V$ that each phase will exhibit a different acoustic reflectivity.

In Fig. 42, we display the optical and acoustic response of another important class of materials. For this sample we coated a silicon wafer with a $1.5-\mu \mathrm{m}$ layer of photoresist. We then exposed this layer with UV light through a mesh in the form of regularly spaced lines. We followed the conventional procedures for photolithography except for one important difference-we did not develop the photoresist after exposure to the UV light. Because of this omission we do not see the pattern in the optical image of Fig. 42(b) since the optical index change is not sufficient. However, the cross linking in the polymer film that does occur after exposure is sufficient 


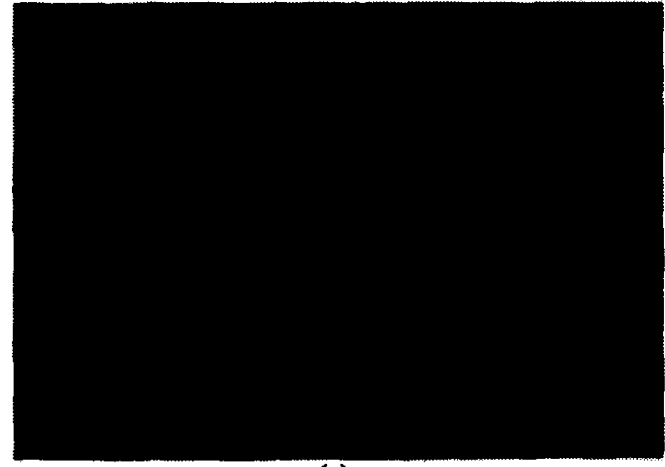

(a)

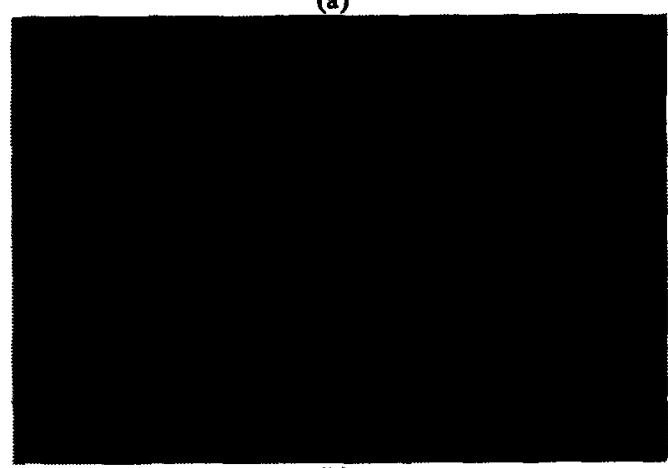

(b)

Fig. 42. Comparison of (a) acoustic and (b) optical images of an exposed but undeveloped pattern in photoresist $(1.5 \mu \mathrm{m})$ on a silicon wafer.

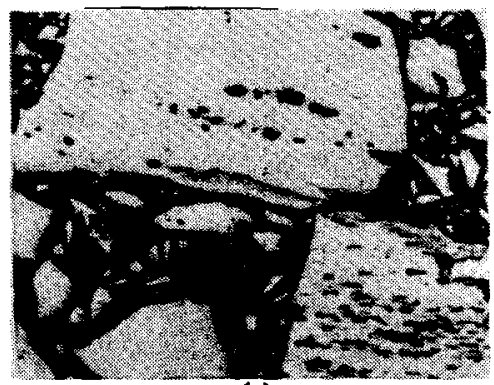

(a)

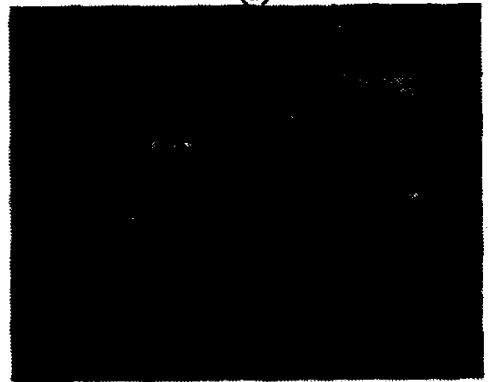

(b)

Fig. 43. (a) Optical $(\times 125)$ and (b) acoustic images in reflection of polished samples of coal. Coal sample from U.S. Steel (medium rank) $f=1100 \mathrm{MHz}$.

to alter the elastic properties to a degree that permits us to see the bar pattern in the acoustic micrograph. We also see certain light splotches in Fig. 39(a). This may be indicative of uneveness in the thickness of the photoresist but we have not explored this in great detail. We believe that this property will be of technological significance in integrated circuits.

In Fig. 43, we show the comparison for an entirely different class of materials-fossil fuel in the form of coal. The important point here is again the increased contrast in the acoustic micrograph. In a recent article in Physics Today reviewing the technology relating to coal it is pointed out that the most ef-

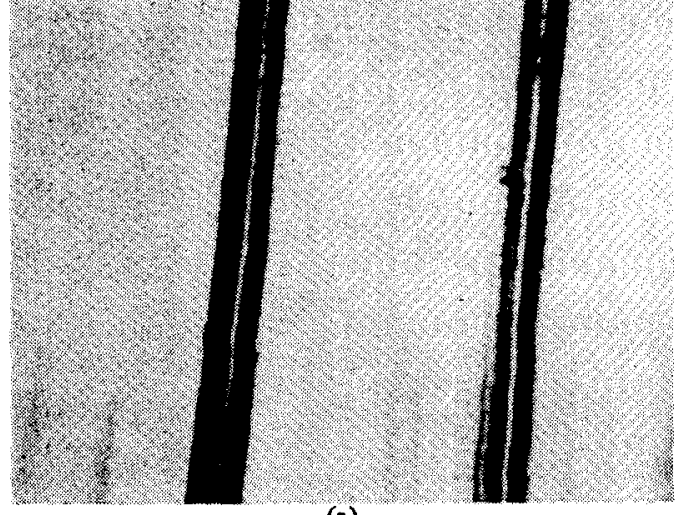

(a)

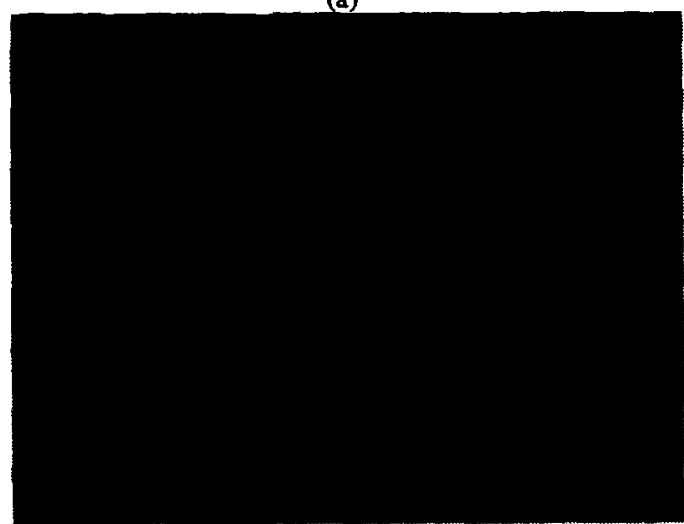

(b)

Fig. 44. Acoustic micrographs of polymer (Keviar) fibers. Transmission at $1400 \mathrm{MHz}$. (a) $\times 500$. (b) $\times 800$.

fective method for determining the carbon content of coal specimen is the measurement of optical reflectivity. Because of the complexity of this material the optical reflectivity as measured point-by-point across the specimen can be calibrated in such a way as to reveal the carbon content. The point-ofinterest here is one of degree. The optical reflectivity varies between 1 and 2 percent and it is between these two limits that the comparison is made. The acoustic reflectivity is much larger-typically 10 to 100 percent-and the variation of reflectivity between the different constituents of the material is greater. This suggests that acoustic microscopy may one day play a role in this field.

Finally, in Fig. 44, we show the acoustic images of polymer textile fibers-Kevlar. This also represents an important class of materials. The various inhomogeneities that show up here are difficult to see in optical images. The changes in elastic parameters appear to be larger than the corresponding changes in the optical index. It could happen that acoustic microscopy will add to the informational content of micrographs in the vast field of fibers.

Now, we turn to a series of integrated circuits--a subject that is probably closer to the professional interest of the readers of Proceedings. We begin with a sample of Fig. 45 that is simplenamely, a single layer of oxide $1.56 \mu \mathrm{m}$ in thickness grown on silicon. There is a pattern in the oxide but it is of secondary interest here. Our primary interest is the large contrast exhibited by this object. This high degree of contrast indicates that we can detect oxide layer thicknesses of a few hundred angstroms.

In Fig. 46 we see an optical image of a MOS transistor fabricated with SOS technology. The cross section of the wafer is shown in the side drawing. In Figs. 47 and 48 we illustrate the 


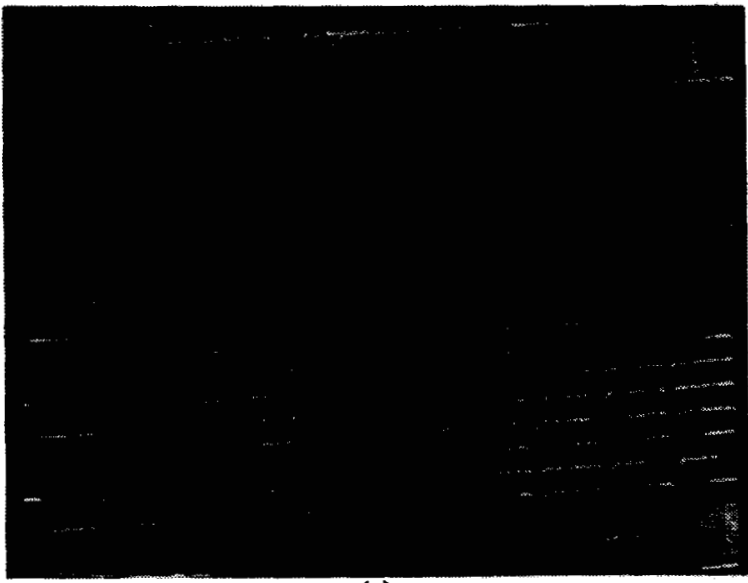

(a)

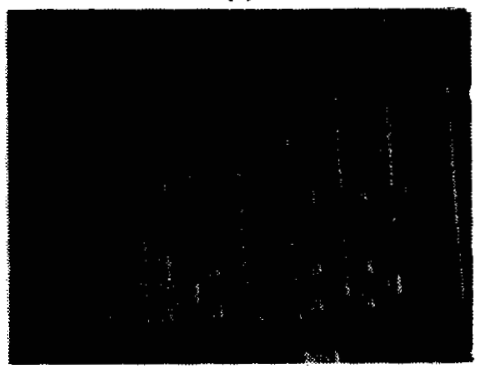

(b)

Fig. 45. Micrographs of silicon wafer coated with oxide. (a) Acoustic $\times 450$. (b) Optical $\times 350$. In (a) dark area has $1.56 \mu \mathrm{m}$ of oxidelight area has $0.35 \mu \mathrm{m}$ of nitride.

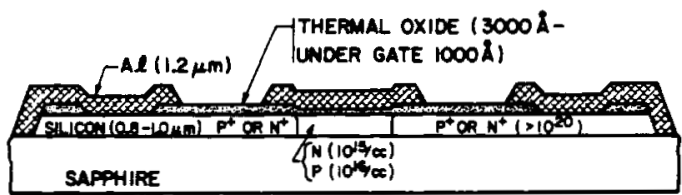

$4000 \mathrm{~A}$ OF LOW DENSITY OXIDE OVER TOP SURFACE

(a)

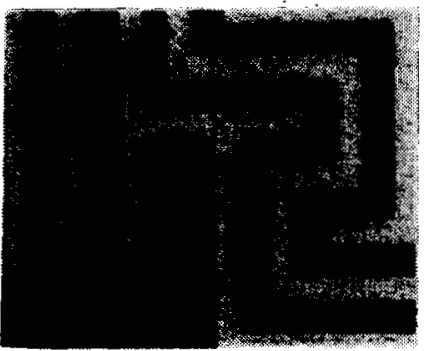

(b)

Fig. 46. Cross sectional view and optical image of an SOS-MOS device. Aluminum linewidth is $7.5 \mu \mathrm{m}$. (a) Optical brightfield by Koch (X1120). (b) (CMOS transistor profile STDL-4 circuit courtesy of Electronics Research Center, Rockwell International.)

appearance of two different devices that were fabricated on the same sapphire wafer. It is instructive to concentrate on the differences between the two devices. It is clearly apparent that the differences in the acoustic micrographs (taken at 1100 $\mathrm{MHz}$ ) are much larger than they are in the optical comparisons. Here again we suggest that the informational content of the two images is greater than that of the optical alone.

If we move now to a higher frequency $(1800 \mathrm{MHz})$ something new appears-namely, the changes in the oxide thickness beneath an aluminum layer becomes apparent as in Fig. 49 [3]. Again it is the SOS circuit with $3000 \AA$ of oxide between the silicon and the aluminum as shown in Fig. 46. But the oxide thins to $1000 \AA$ at the gate regions and these thinned regions

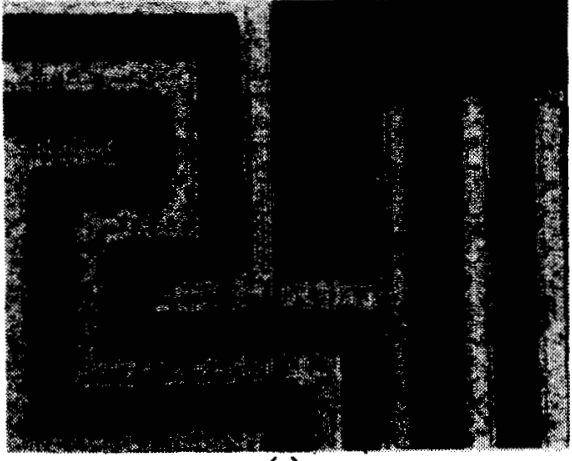

(a)

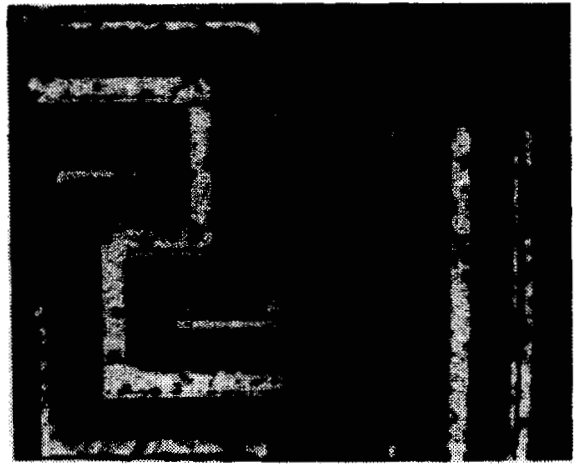

(b)

Fig. 47. Optical and acoustic comparison of a CMOS device with SOS technology. (a) Optical brightfield by Koch (X1120). (b) Acoustic $f=1100 \mathrm{MHz}$ (SOS chip B-STDL-4, Rockwell CMOS).

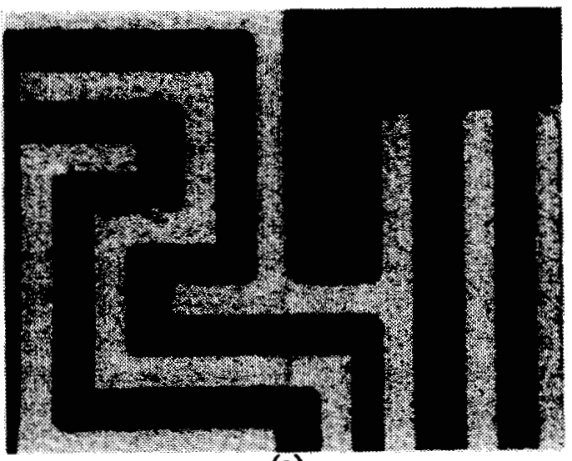

a

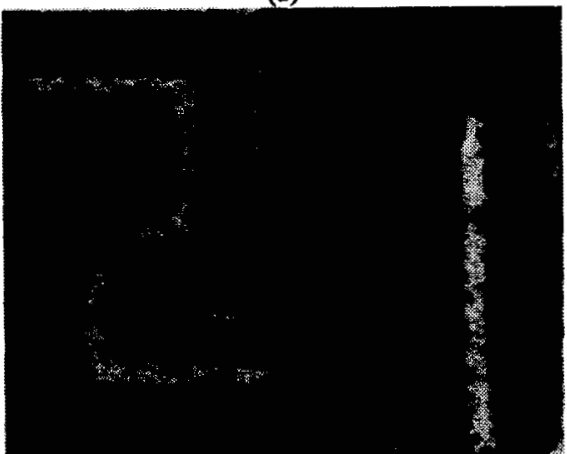

(b)

Fig 48. A second CMOS device on the same wafer as that shown in Fig. 47. (a) Optical interference contrast by Koch $(\times 1120)$. (b) Acoustic $f=1100 \mathrm{MHz}$ (SOS chip A-STDL-4, Rockwell CMOS).

are evident in the acoustic micrograph of Fig. 49(b). In this image and the previous two, the linewidth is $7.5 \mu \mathrm{m}$. In Fig. 50 we have the appearance of a circuit as fabricated on silicon [3]. The aluminum linewidth here is $5 \mu \mathrm{m}$ and we can identify a number of differences between the optical and the 


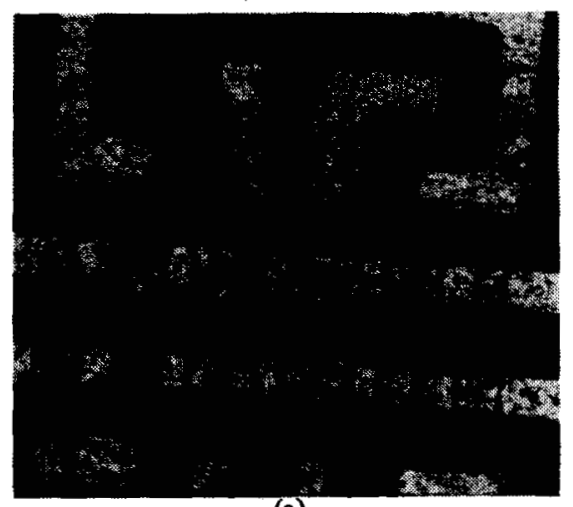

(a)

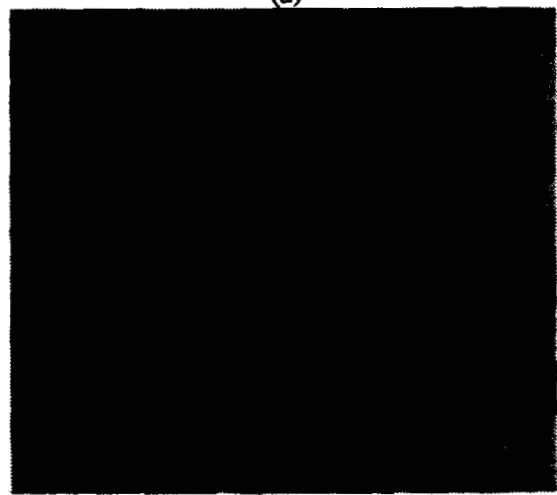

(b)

Fig. 49. CMOS device showing cross overs (upper aluminum lines) and gates (right and left devices on lower aluminum line). (a) Optical bright field. (b) Acoustic-1800 MHz. (Courtesy of V. Jipson.)

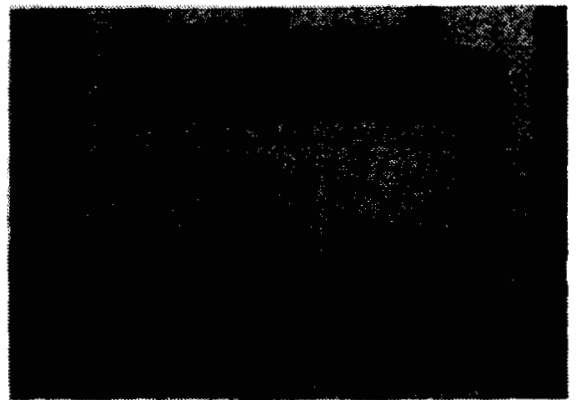

(a)

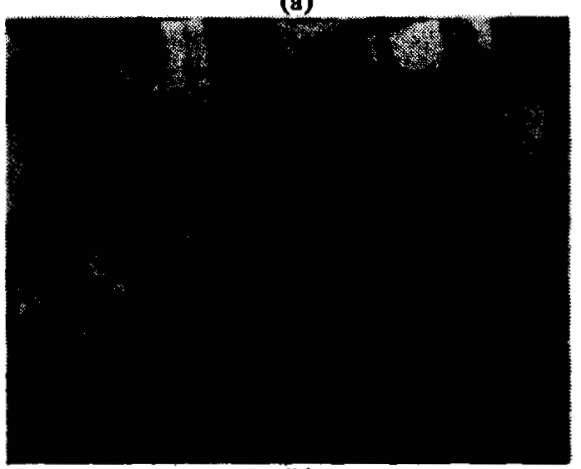

(b)

Fig. 50. Comparison of the optical (a) and acoustic (b) images of a different region of the same wafer as Fig. 47. Of interest are the dark blotches which appear on the aluminum lines in the acoustic micrograph but are not visible on the optical image (Jipson [3]).

acoustic images. We believe some of the differences are a result of inhomogeneities in the elastic properties of the devices.

The final image of Fig. 51 is placed here to exhibit the micrographs of GaAs devices and to illustrate in a graphic way the

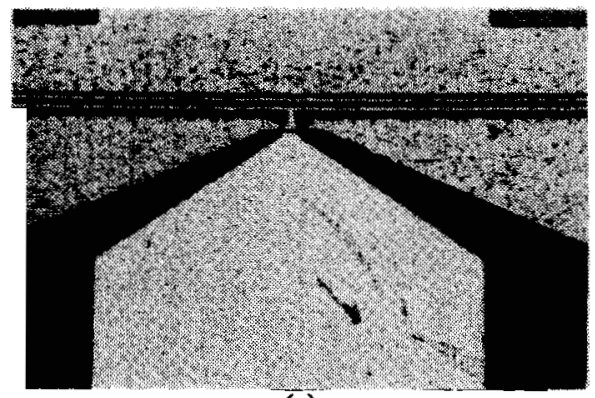

(a)

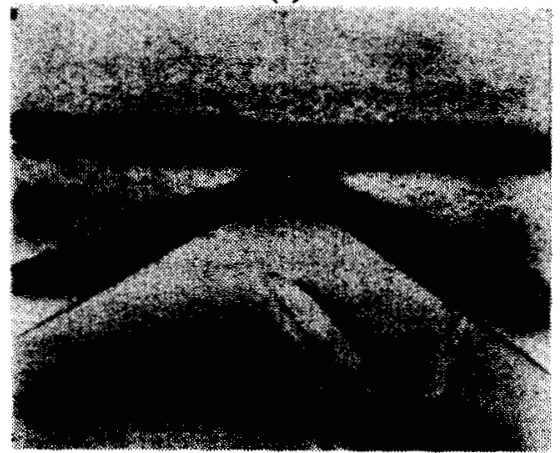

(b)

Fig. 51. Comparison of the optical (a) and acoustic (b) images of a dual gate GaAs FET. The gate lines are $1 \mu \mathrm{m}$ wide with a $1 \mu \mathrm{m}$ spacing (Jipson [3]).

resolution that is now possible with acoustic microscopy. This dual gate FET is made with 1 linewidth spaced by $1 \mu \mathrm{m}$. We can see that these lines are easily resolved in both micrographs, and, again, there is no lack of contrast in the acoustic micrographs.

\section{SUMMARY}

In this review we have tried to demonstrate that acoustics can compete in a number of ways with optics. They represent a new method for viewing microscopic objects. Acoustic radiation is a viable alternative to more conventional microscopy, and in numerous problem areas it can be used to complement and extend the optical instrument. The elastic properties as monitored on a microscopic scale are important parameters that enter into structures over a range from biological cells, to fossil fuels (particularly coal), to materials such as steel, important in technology through the fabricated microstructures in the field of microelectronics.

We have pointed out that the state of the art has been advanced in the past year. The wavelength used in the best instrument is now equal to that of green light. The theory that is needed to explain the high contrast and the variations in contrast has been worked out. It is now possible to understand a good deal of the information that appears in the images.

We also hold that there is a promising future for a microscope which combines an optical input with an acoustic output-a photoacoustic microscope. In this system the optical input is a laser modulated in amplitude at a microwave frequency. The thermal expansion coefficient of the object produces strains at this modulation frequency. These optically induced strains are to be detected with the acoustic lens and the output transducer. Progress on this suggestion has been intermittent, and for that reason, it is more appropriate to wait for a future review.

We want to leave the reader with the impression that there is still more to come. Shorter wavelengths will be used with a resulting increase in resolving power. Signal processing routines 
used to enhance the images are evolving, and a dedicated computer to manipulate the electrical signals used to form the image will extend the power of this instrument far beyond what is suggested here. The realm of cryogenic liquids holds great promise, and it should allow us to use wavelengths in the submicrometer region below the optical limit.

We predict the new advances will occur on three frontsroom temperature liquids, cryogenic liquids, and photoacoustics. We end this review with that as our hopeful outlook for the future of acoustic microscopy.

\section{ACKNOWLEDGMENT}

A number of people have contributed to the progress in acoustic microscopy as reported here. They have participated both directly and in discussion sessions. Their time was freely given, and we want to express our strong appreciation for their enthusiasm. In the Ginzton Laboratory, M. Chodorow, G. Kino, and the late R. Kompfner have made constructive suggestions since the beginning of the project. At Zenith Research Laboratories, A. Korpel and L. Kessler have shared their ideas on acoustic microscopy with us. $\mathbf{R}$. Wilson and $\mathbf{R}$. Weglein of Hughes Research Laboratories have contributed a number of ideas that will continue to be important. M. Bullis at the National Bureau of Standards and E. Ash at University College London encouraged us to continue in the early stages when the images were lacking in significant detail.

In our own laboratories, the work of $\mathrm{R}$. Lemons has already been referenced and we only need add that he ground the first lens with his own hands. The late W. Bond did much of the early design work and the instrument was constructed by $G$. Bicker. L. Goddard has shared his expertise with us throughout the program and the performance of the acoustic components is due to his careful work. G. Kotler and J. Vrhel provided the sapphire crystals with precision surfaces and spherical lenses which form the heart of the entire system. V. Jipson set about to improve the resolution and he has shared his high-quality images with us. J. Heiserman has contributed to all aspects of the work with biological specimens. Ms. R. Koch, a member of the Center for Materials Research, has contributed the high quality optical images. Ms. N. Pleibel, of the Cancer Biology Research Laboratory, has provided us with many of the biological cells. We also want to express our appreciation for the samples that we have received from a number of interested friends. These include P. J. Hagon of Rockwell International (the SOS samples), C. Liechti of Hewlett-Packard (the GaAs FET samples), R. Grey of the U.S. Steel Research Laboratory, for suggesting the studies of coal, and P. Tucker of the School of Textiles, North Carolina State University, Raleigh, for suggesting the study of polymer fibers.

\section{REFERENCES}

[1] J. Ilukor and E. H. Jacobsen, "Coherent elastic wave propagation in quartz at ultramicrowave frequencies," in Physical Acoustics, W. P. Mason, Ed. New York: Academic Press, 1968, vol. V, ch. 5, pp. 221-231; and C. H. Anderson and E. S. Sabisky, "Spinphonon spectrometer," in Physical Acoustics, W. P. Mason and R. N. Thurston, Eds. New York: Academic Press, 1971, vol. VIII, ch. 1, pp. 2-57.

[2] J. S. Imai and I. Rudnick, "U1trasonic at tenuation in liquid helium at 1 GHz," Phys. Rev. Lett., vol. 22, pp. 694-697, Apr. 7, 1969.

[3] V. Jipson and C. F. Quate, "Acoustic microscopy at optical wavelengths," Appl. Phys. Lett., vol. 32, pp. 789-791, June 15, 1978.

[4] R. A. Lemons and C. F. Quate, "Acoustic microscopy: Biomedical applications," Science, vol. 188, pp. 905-911, May 30, 1975, and $R$. Kompfner and C. F. Quate, "Acoustic radiation and its use in microscopy," Phys. Technol., vol. 8, pp. 231-237, Nov. 1977.
[5] A. Korpel, "Acoustic microscopy," in Ultrasonic Imaging and Holography, G. W. Stroke et al., Eds. New York: Plenum Press, 1974.

[6] L. W. Kessler, "A review of progress and applications in acoustic microscopy," J. Acoust. Soc. Amer., vol. 55, pp. 909-918, May 1974.

[7] N. Chubachi, "Acoustic microscopy-A review," Oyo Butsuri, vol. 47, p. 75, Apr. 1978 (in Japanese).

[8] C. F. Quate, "Imaging using lenses," in Acoustic Imaging: Cameras, Microscopes, Phased Arrays and Holographic Systems, G. Wade, Ed. New York: Plenum Press, 1976, ch. 11, pp. 241-305.

[9] R. A. Lemons and C. F. Quate, "Acoustic microscopy," in Physical Acoustics, vol. XIV, R. N. Thurston, Ed. New York: Academic Press, to be published.

[10] L. W. Kessler and D. E. Yuhas, "Acoustic microscopy-1979," Proc. IEEE, vol. 67, pp. 526-535, Apr. 1979.

[11] T. G. Rochow and E. G. Rochow, An Introduction to Microscopy by Means of Light, Electrons, X-Rays, or Ultrasound. New York: Plenum Press, 1978.

[12] L. W. Kessler, P. R. Palermo, and A. Korpel, "Recent developments with the scanning laser acoustic microscope," in Acoustical Holography, P. S. Green, Ed. New York: Plenum Press, 1974, pp. 15-23; and A. Madeyski and L. W. Kessler, "Initial experiments in the application of acoustic microscopy to the characterization of steel and to the study of fracture phenomena," IEEE Trans. Sonics-Ultrason., vol. SU-23, pp. 363-369, Sept. 1976.

[13] L. W. Kessler and D. E. Yuhas, "Structural perspective," Indus. Res, vol. 20 , pp. 53-56, Jan. 1978.

[14] C. S. Tsai, S. K. Wang, and C. C. Lee, "Visualization of solid material joints using a transmission-type scanning acoustic microscope," Appl. Phys. Lett., vol. 31, pp. 317-320, Sept. 1, 1977.

[15] R. G. Wilson, R. D. Weglein, and D. M. Bonnel, in Semiconductor Silicon $/ 1977$, H. R. Huff and E. Sirtl, Eds. Princeton, NJ: Electrochem. Soc., vol. 77, no. 2, pp. 431-440, 1977.

[16] R. D. Weglein and R. G. Wilson, "Characteristic material signatures by acoustic microscopy," Electron. Lett., vol. 14, pp. 352-354, June 8, 1978.

[17] B. Bridoux et al. "Optimization of a transmission acoustic microscope," J. Appl. Phys., vol. 49, pp. 574-579, Feb. 1978.

[18] J. Attal and G. Cambon, "Signal processing in the reflective acoustic microscope," Electron. Lett., vol. 14, pp. 472-473, July 20, 1978.

[19] H. K. Wickramasinghe and M. Hall, "Phase imaging with the scanning acoustic microscope," Electron. Lett., vol. 12, pp. 637-638, Nov. 25, 1976.

[20] N. Chubachi, T. Sannomiya, and T. Iyama, "Scanning acoustic microscope with concave transducers," presented at Joint Meeting, Acoustical Society of America and Acoustical Society of Japan, Honolulu, HI, Nov. 27-Dec. 1, 1978.

[21] S. Bennett, D. Payne, and E. A. Ash, "Reflective geometry for microscopy and NDT," in 1977 Ultrasonics Symp. Proc., J. deKlerk and B. R. McAvoy, Eds. (IEEE Cat. No. $77 \mathrm{CH} 1264$ 1SU), pp. 161-164.

[22] A. F. Brown, "Seeing with sound," Endeavour, vol. 35, pp. 123-128, Sept. 1976.

[23] R. C. Eggleton, "Application of acoustic microscopy to the study of muscle mechanics," in Multidisciplinary Microscopy, SPIE Proc. vol. 104, R. L. Whitman, Ed. Bellingham, WA 98225: Soc. Photo-Optical Instrumentation Engineers (P.O. Box 10), pp. 117-1 24, 1977.

[24] G. Larsen, "Det Akustiske Mikroskop," Saetrykk av Farmako. terapi, vol. 33, pp. 49-55, 1977 (in Norwegian).

[25] L. Broomhead, "La microscopie acoustique," Sci. A venir, pp. 71-77, May 1978 (in French).

[26] T. H. Maugh, II, "Acoustic microscopy: A new window to the world of the small," Science, vol. 201, pp. 1110-1114, Sept. 22, 1978.

[27] T. M. Reeder and D. K. Winslow, "Characteristics of microwave acoustic transducers for volume wave excitation," IEEE Trans. Microwave Theory Tech., vol. MTT-17, pp. 927-942, Nov. 1969.

[28] R. A. Lemons, "Acoustic microscopy by mechanical scanning," Ph.D. dissertation, Stanford Univ., Stanford, CA, May 1975, unpublished.

[29] R. D. Weglein, "Acoustic properties of sputtered glass at microwave frequencies," Appl. Phys. Lett., vol. 29, pp. 277-279, Sept. $1,1976$.

[30] Further details of the lens design and some of the electronic circuitry can be found in the Annu. Rep. for NSF/RANN, NTIS PB271443, Apr. 1977.

[31] R. Kompfner and R. A. Lemons, "Nonlinear acoustic microscopy," Appl. Phys. Lett., vol. 28, pp. 295-297, Mar. 15, 1976; and $H$. K. Wickramasinghe and $C$. Yeack, "Nonlinear imaging of an edge in the scanning acoustic microscope," J. Appl. Phys., vol. 48, pp. 4951-4954, Dec. 1977.

[32] W. L. Bond et al., "Dark field and stereo viewing with the acoustic microscope," Appl. Phys. Lett., vol. 27, pp. 270-272, Sept. 1, 1975. 
[33] K. F. Hertzfeld and T. A. Litovitz, Absorption and Dispersion of Ultresonic Waves. New York: Academic Press, 1959.

[34] M. T. Wauk, II, "Attenuation in microwave transducers and resonators," Ph.D. dissertation, Stanford University, Stanford, CA, July 1969 , unpublished.

[35] A. Akhieser, "On the absorption of sound in solids," $J$. Phys. (Aka de miia Nauk-Leningrad), vol. 1, pp. 277-287, 1939.

[36] I. L. Fabelinskii, Molecular Scattering of Light. New York: Plenum Press, 1968.

[37] T. O. Woodruff and $H$. Ehrenreich, "Absorption of sound in insulators," Phys. Rev., vol. 123, pp. 1553-1559, Sept. 1, 1961.

[38] J. Attal and C. F. Quate, "Investigation of some low ultrasonic absorption liquids," J. Acoust. Soc. Amer., vol. 59, pp. 69-73, Jan. 1976.

[39] W. T. Cathey, Optical Information Processing and Holography. New York: Wiley, 1974.

[40] J. W. Goodman, Introduction to Fourier Optics. New York: McGraw-Hill, 1968.

[41] E. O'Neill, Introduction to Statistical Optics. Reading, MA: Addison-Wesley, 1963.

[42] J. A. Ratcliffe, Reports on Progress in Physics, vol. XIX, A. C. Strickland, Ed. London, England: The Physical Society, 1956.

[43] D. C. Champeney, Fourier Transforms and Their Physical Appli. cations. New York: Academic Press, 1973.

[44] -, Loc. cit. Section 11.5.

[45] H. K. Wickramasinghe, "Contrast and imaging performance in the scanning acoustic microscope," J. Appl. Phys., vol. 50, pp. 664-672, Feb. 1979.

[46] E. Evans and Y. C. Fung, "Improved measurements of the erythrocyte geometry," Microvas. Res., vol. 4, pp. 335-347, 1972.

[47] J. W. Goodman, loc. cit. Section 6.4.

[48] A. Atalar, "An angular-spectrurn approach to contrast in reflection acoustic microscopy," J. Appl. Phys., vol. 49, pp. 51305139 , Oct. 1978.

[49] H. K. Wickramasinghe, "Contrast in reflection acoustic microscopy," Electron. Lett., vol. 14, pp. 305-306, May 11, 1978.

[50] A. Atalar, C.F. Quate, and H. K. Wickramasinghe, "Phase imaging in reflection with the acoustic microscope," Appl. Phys. Lett., vol. 31, pp. 791-793, Dec. 15, 1977.

[51] L. M. Brekhovskikh, Waves in Layered Media. New York: Academic Press, 1960, pp. 100-122.

[52] A. Atalar, "Reflection coefticient for a lossy liquid-lossless isotropic solid interface," J. Acoust. Soc. Amer., June 1979.

[53] M. F. Marmor, H. K. Wickramasinghe, and R. A. Lemons, "Acoustic microscopy of the human retina and pigment epithelium," Investiga. Ophthalmol. Vis. Sci., vol. 16, pp. 660-666, July 1977. 


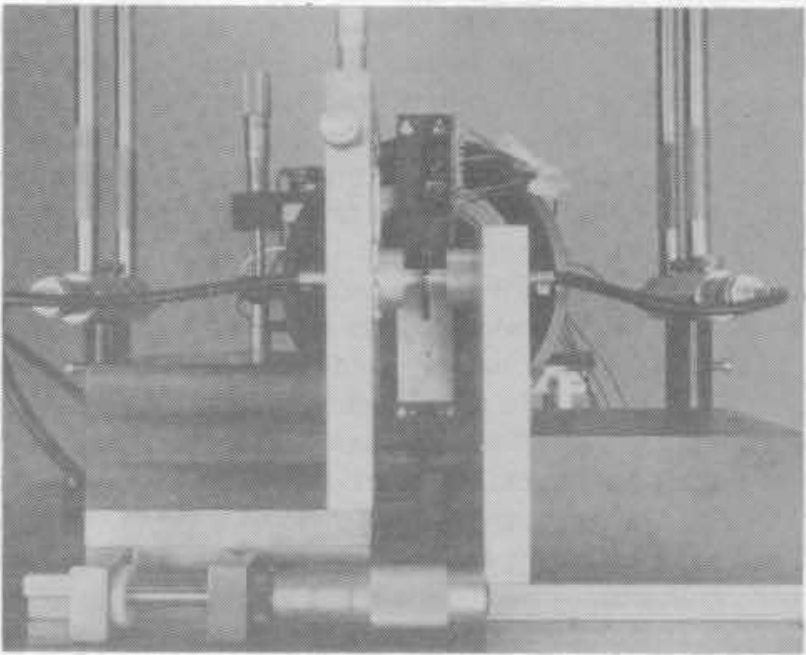

Fig. 3. Scanning acoustic microscope-Mechanical components. (Courtesy of R. C. Addison.) 


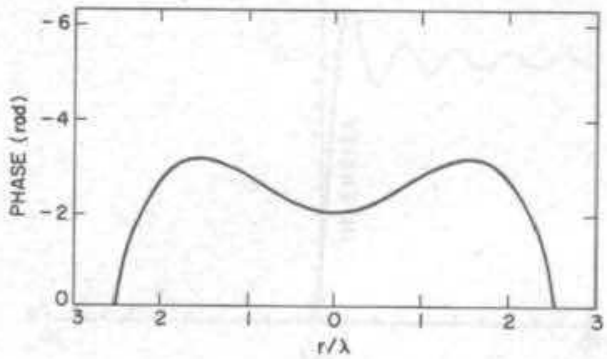

(a)

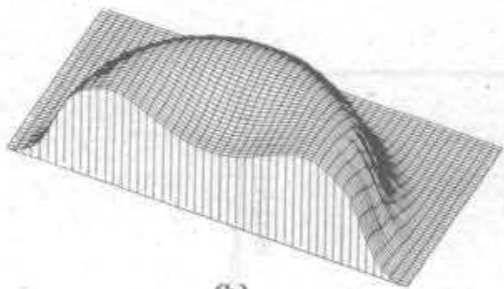

(b)

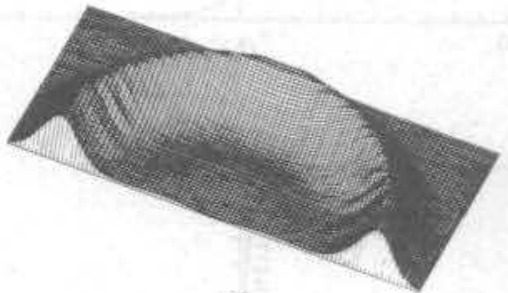

(c)

Fig. 18, Ideal red blood cells. (a) Phase profile from Evans and Fung [46] (hemoglobin absorption equal to water). (b) Phase response of transducer output. Grid lines are $\lambda / 8$ by $\lambda / 16$. (c) Amplitude response of transducer together with the derivative of the phase responses in (b). Grid lines are $\lambda / 16$ by $\lambda / 32$.

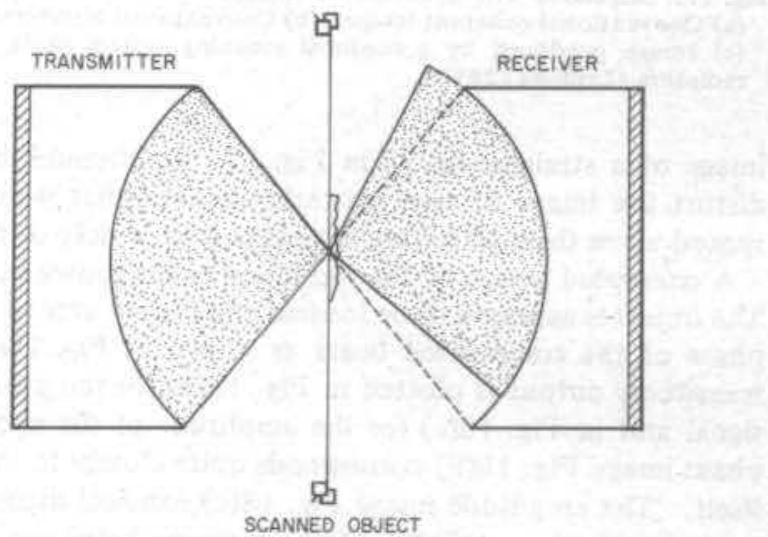

Fig. 19. Illustration of change in illumination of the lens when the beam encounters a transverse phase gradient in the focal plane. 
PHASE

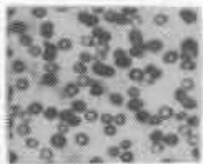

(a)

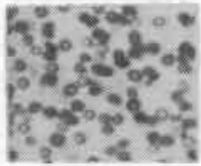

(b)

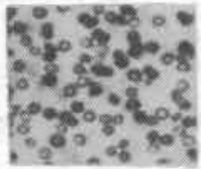

(c)
AMPLITUDE
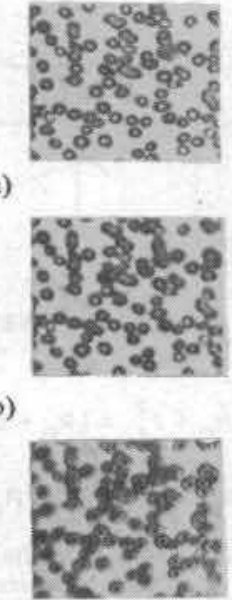

Fig, 22. Experimental images of red blood cells for the three conditions specified in Fig. 20. Note the reversal of contrast in amplitude for (c) the favorable comparison with the theory in Fig. 21(c). 


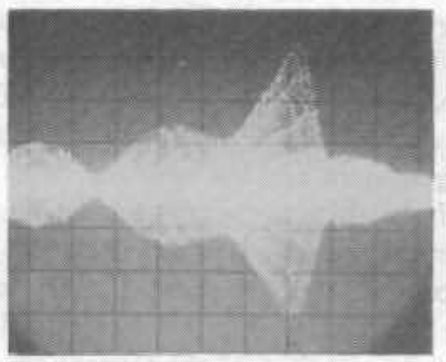

\section{YAG}

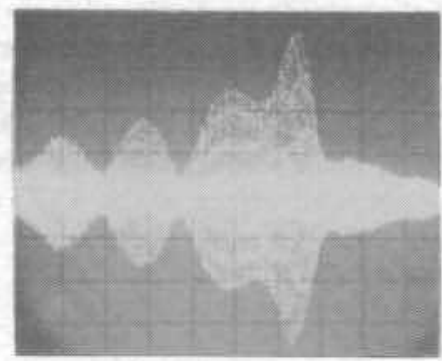

YIG

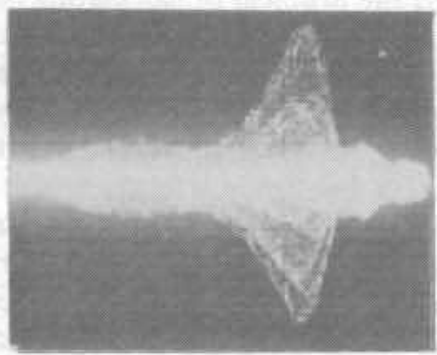

$\mathrm{Al}_{2} \mathrm{O}_{3}$

Fig. 32. Long exposure oscilloscope photos of the returning pulses as the distance between the sample and lens is varied. Envelopes show $V(Z)$ curves for different crystals. Horizontal scale $3.75 \mu \mathrm{m} /$ $\operatorname{div}(1100 \mathrm{MHz})$. 


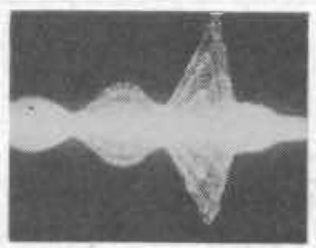

Si

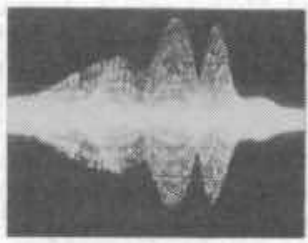

Inm of

A) ON Si

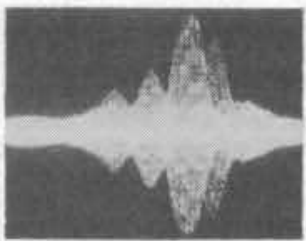

$2 \mu \mathrm{m}$ OF

Al ON Si

Fig. 34. $V(Z)$ photographs for silicon with different thicknesses of aluminum deposited on it. 


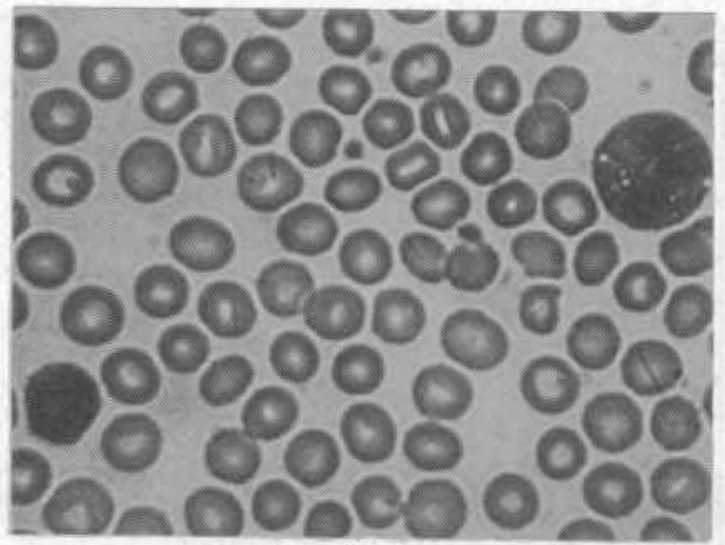

(a)

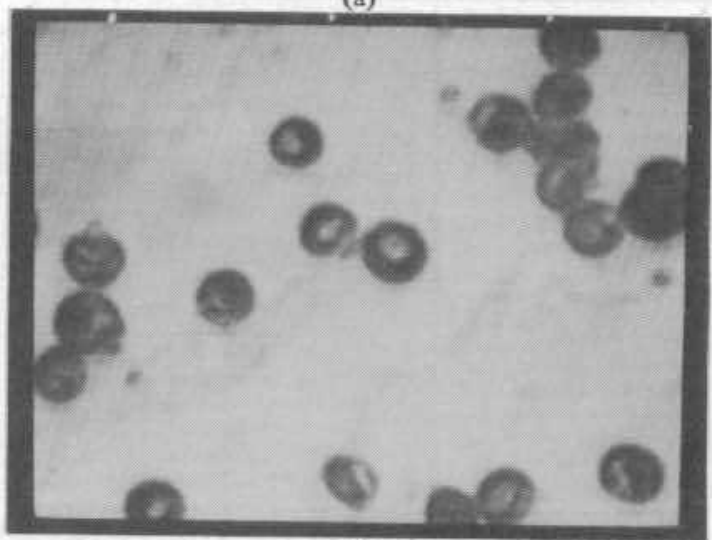

(b)

Fig. 38. Normal red blood cells. (a) Optical fixed in methanol with Leishman's stain (X1000 (oil immersion)). (b) Acoustic (1100 MHz) fixed in methanol, unstained, but mounted on silicon wafer with 1.56 $\mu \mathrm{m}$ of $\mathrm{SiO}_{1}(\times 1000)$. Cells are from different individuals. (Courtesy of J. Heiserman.)

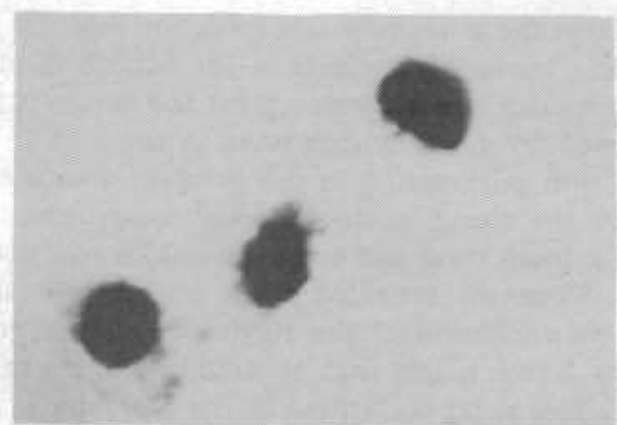

(a)

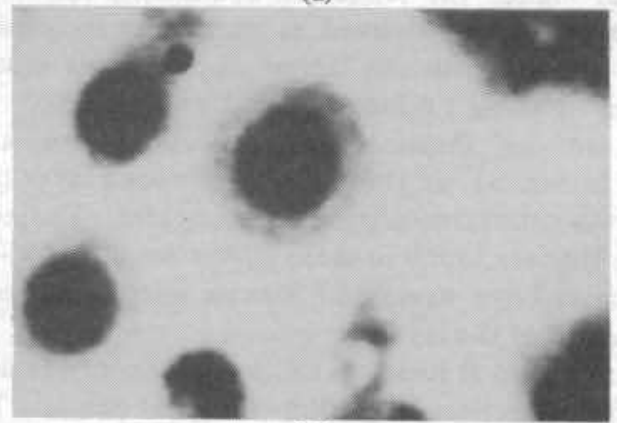

(b)

Fig. 39. Acoustic images in reflection $(1100 \mathrm{MHz})$-Living macrophage cells. 


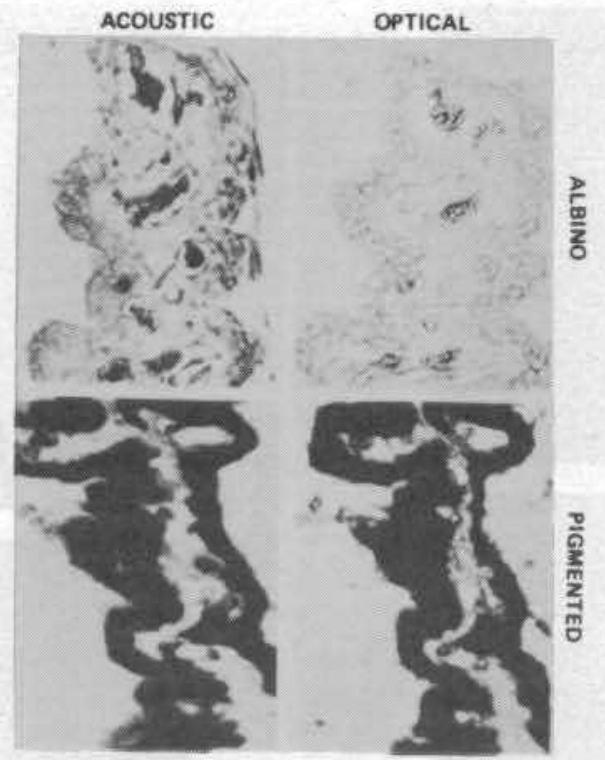

Fig. 40. Sections of iris from an albino and pigmented rabbit (Marmor [53]).
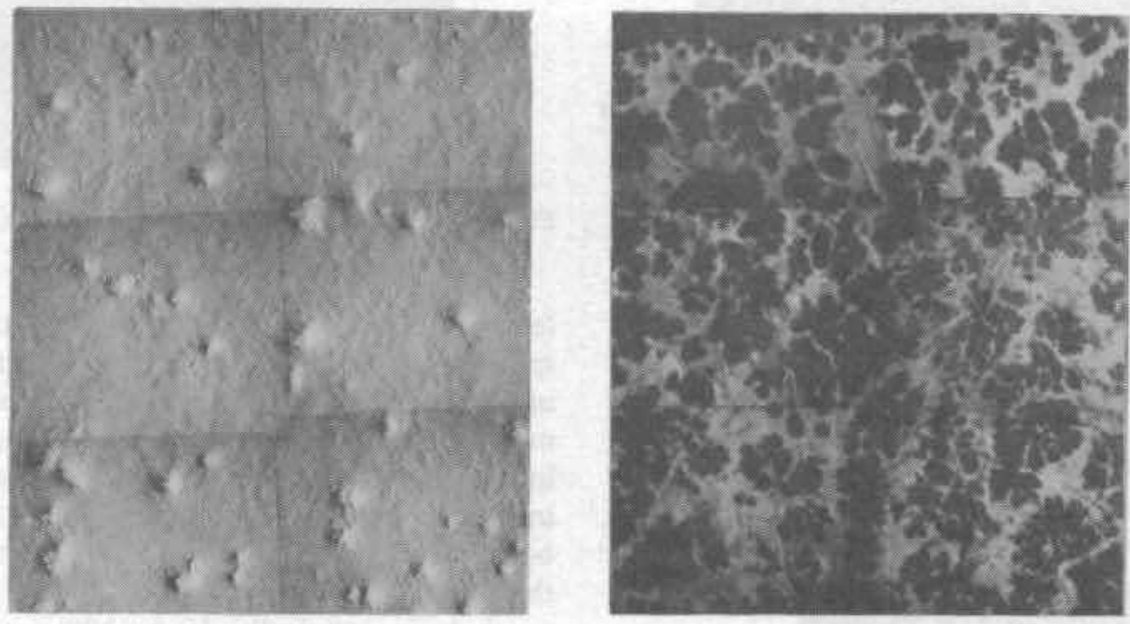

(a)

Fig. 41. Comparison of optical and acoustic images of a Co-Ti alloy. (a) Optical D/C. (b) Acoustic. Co-Ti alloy showing 4 phases. Overall composition 59-percent Co, 41-percent Ti. 


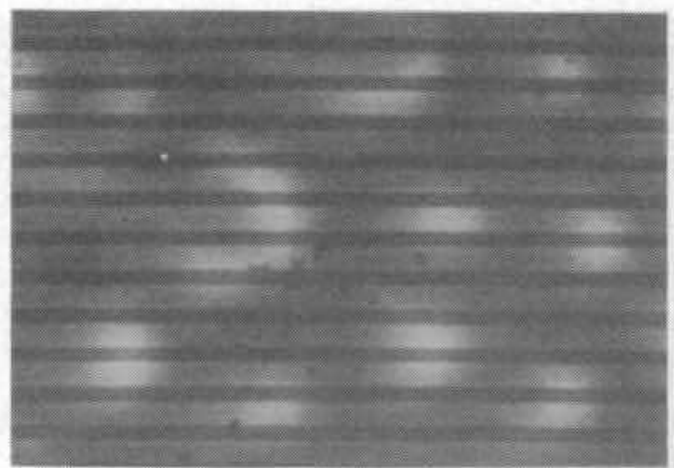

(a)

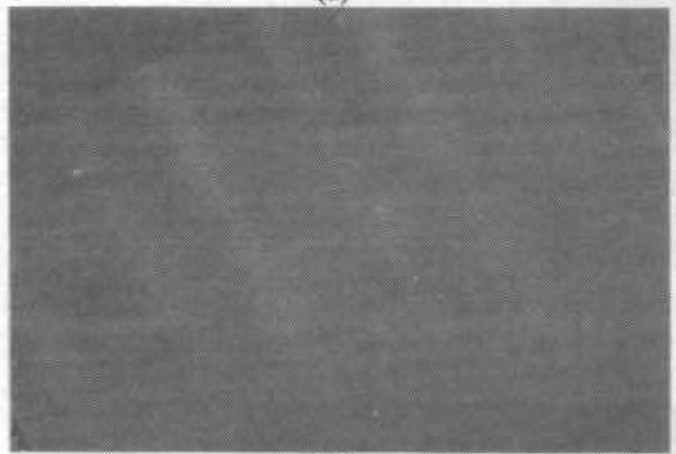

(b)

Fig. 42. Comparison of (a) acoustic and (b) optical images of an exposed but undeveloped pattern in photoresist $(1.5 \mu \mathrm{m})$ on a silicon wafer.

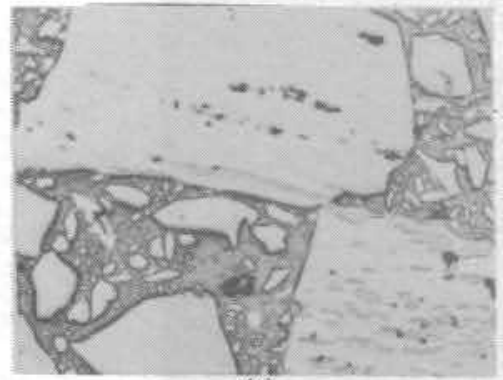

(a)

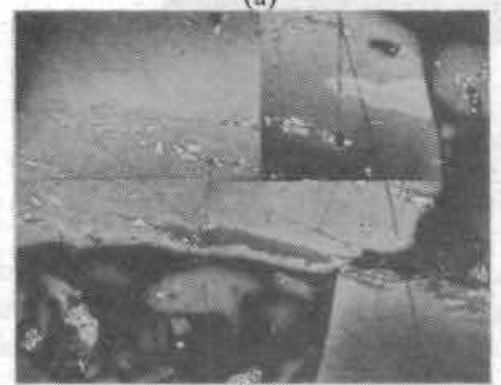

(b)

Fig. 43. (a) Optical $(\times 125)$ and (b) acoustic images in reflection of polished samples of coal. Coal sample from U.S. Steel (medium rank) $f=1100 \mathrm{MHz}$. 


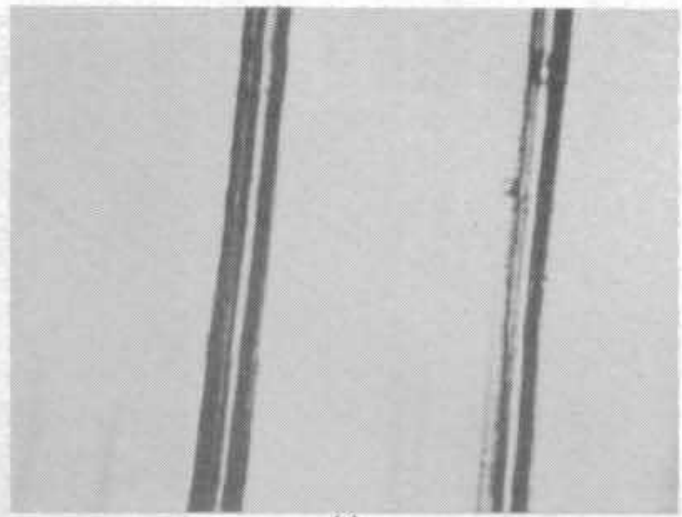

(a)

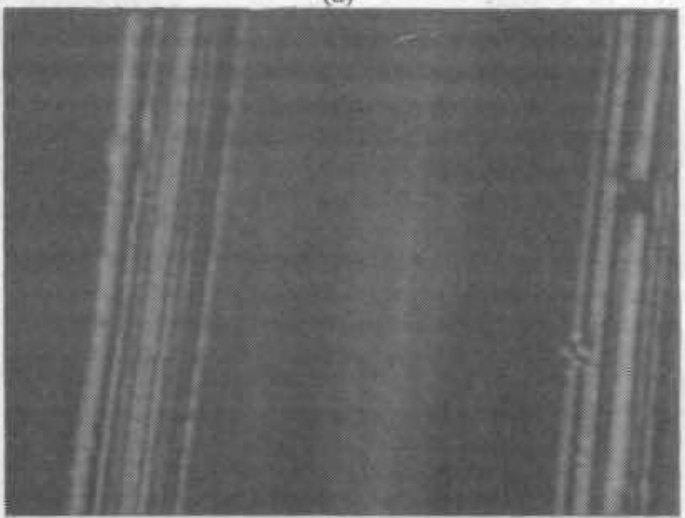

(b)

Fig. 44. Acoustic micrographs of polymer (Kevlar) fibers. Transmission at $1400 \mathrm{MHz}$. (a) $\times 500$. (b) $\times 800$. 


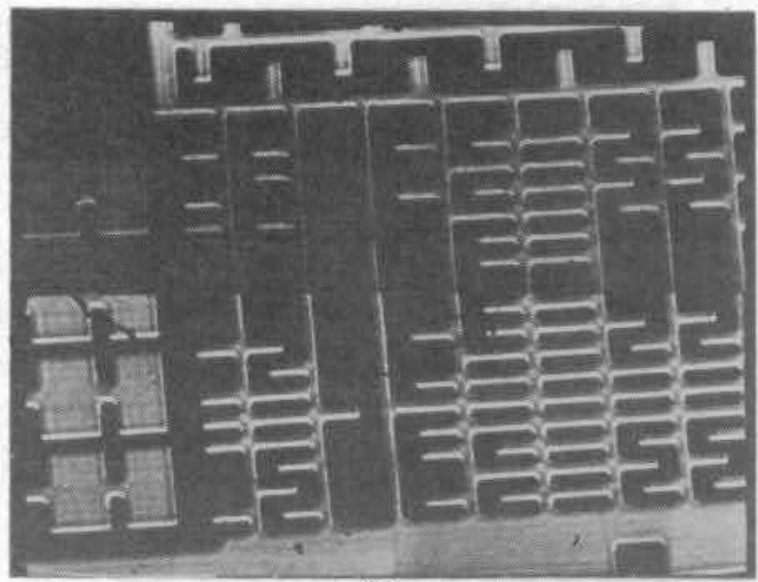

(a)

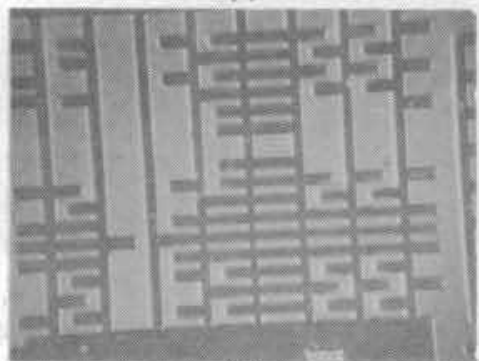

(b)

Fig. 45. Micrographs of silicon wafer coated with oxide. (a) Acoustic $\times 450$. (b) Optical $\times 350$. In (a) dark area has $1.56 \mu \mathrm{m}$ of oxidelight area has $0.35 \mu \mathrm{m}$ of nitride.

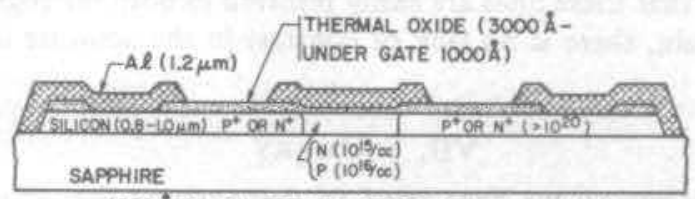

$4000 \AA$ OF LOW DENSITY OXIDE OVER TOP SURFACE

(a)

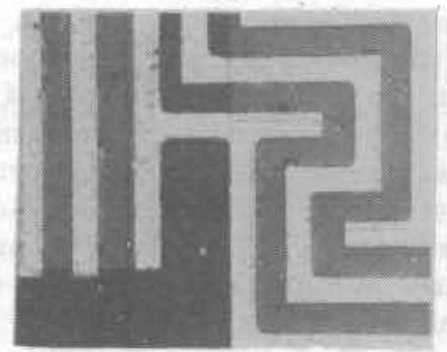

(b)

Fig. 46. Cross sectional view and optical image of an SOS-MOS device. Aluminum linewidth is $7.5 \mu \mathrm{m}$. (a) Optical brightfield by Koch (X1120). (b) (CMOS transistor profile STDL-4 circuit courtesy of Electronics Research Center, Rockwell International.) 


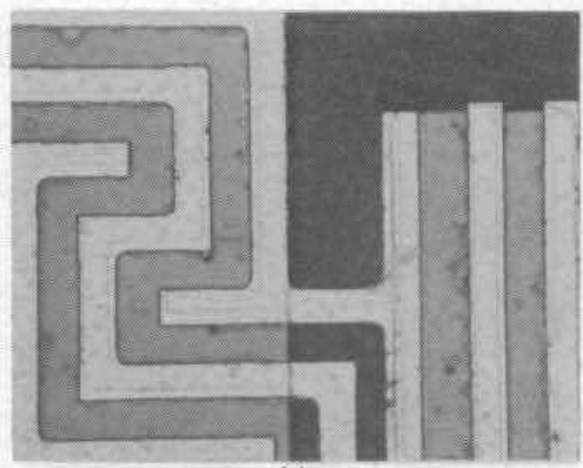

(a)

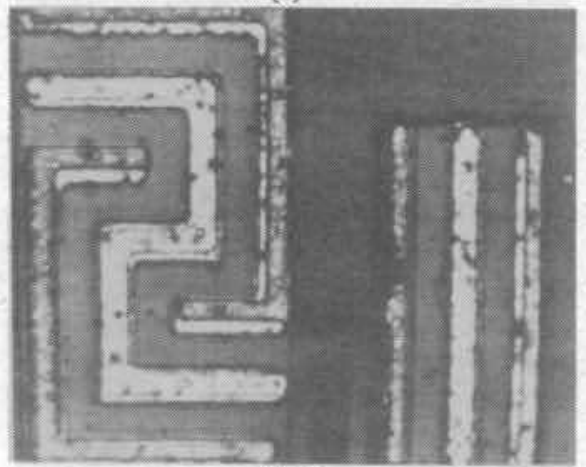

(b)

Fig. 47. Optical and acoustic comparison of a CMOS device with SOS technology. (a) Optical brightfield by Koch $\left(X_{1120}\right)$. (b) Acoustic $f=1100 \mathrm{MHz}$ (SOS chip B-STDL-4, Rockwell CMOS).

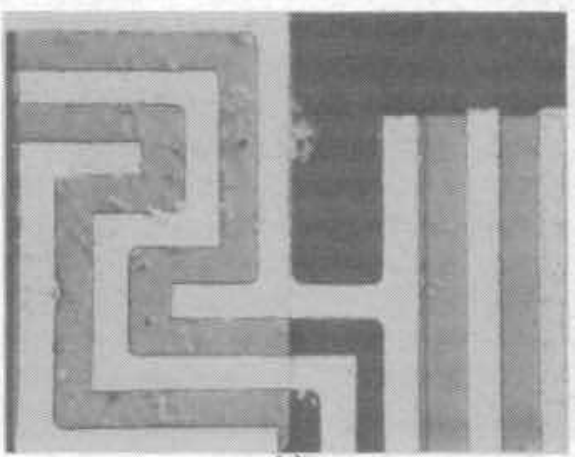

(a)

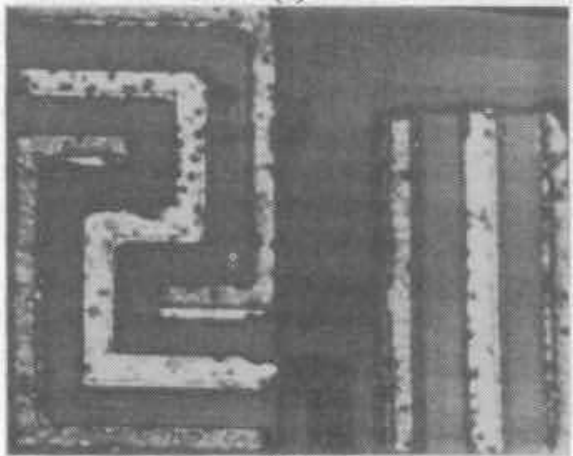

(b)

Fig. 48. A second CMOS device on the same wafer as that shown in Fig. 47. (a) Optical interference contrast by Koch $\left(X_{1120}\right)$. (b) Acoustic $f=1100 \mathrm{MHz}$ (SOS chip A-STDL-4, Rockwell CMOS). 


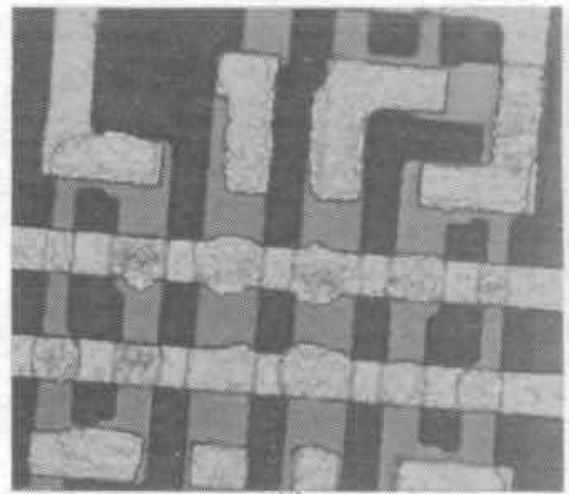

(a)

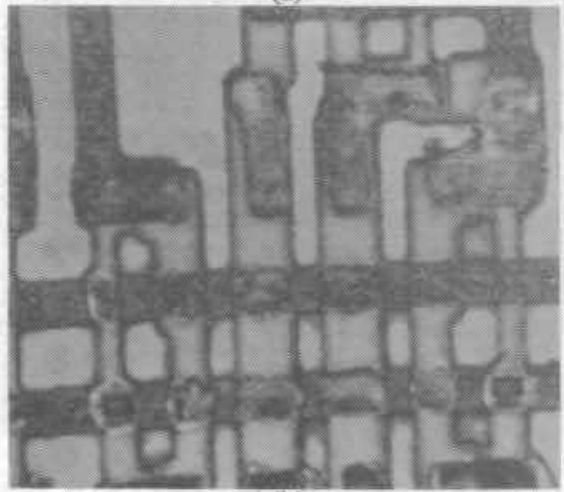

(b)

Fig. 49. CMOS device showing cross overs (upper aluminum lines) and gates (right and left devices on lower aluminum line). (a) Optical bright field. (b) Acoustic $-1800 \mathrm{MHz}$. (Courtesy of V. Jipson.)

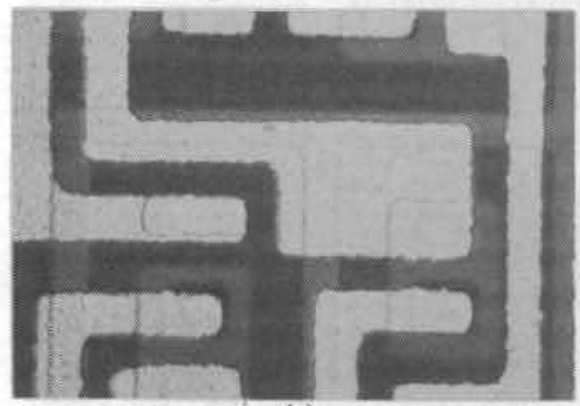

(a)

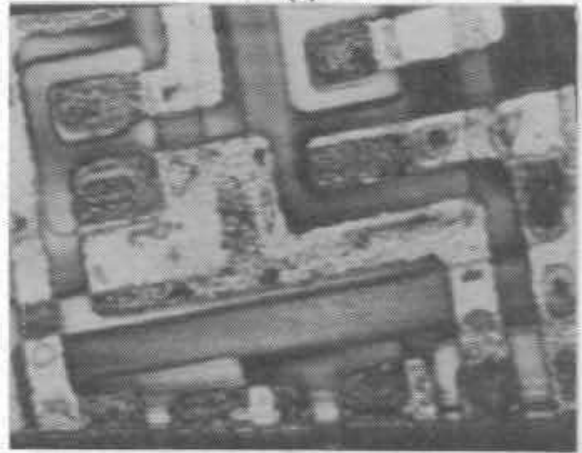

(b)

Fig. 50. Comparison of the optical (a) and acoustic (b) images of a different region of the same wafer as Fig. 47 . Of interest are the dark blotches which appear on the aluminum lines in the acoustic micrograph but are not visible on the optical image (Jipson [3]). 


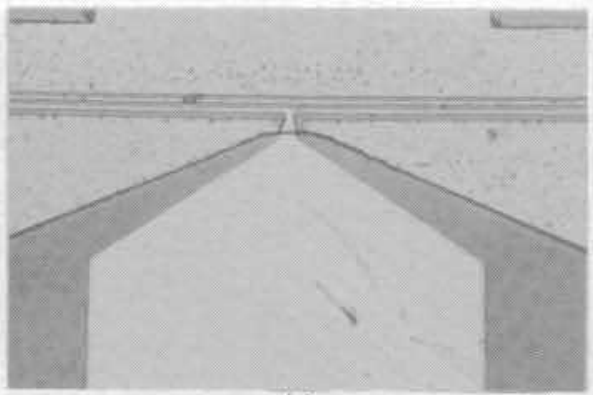

(a)

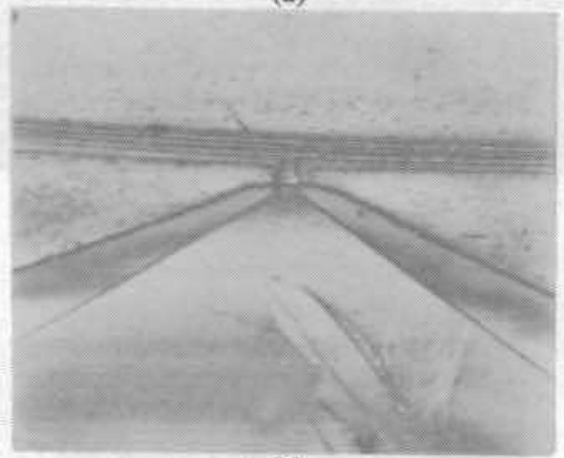

(b)

Fig. 51. Comparison of the optical (a) and acoustic (b) images of a dual gate GaAs FET. The gate lines are $1 \mu \mathrm{m}$ wide with a $1 \mu \mathrm{m}$ spacing (Jipson [3]). 